Copyright (C) 2009. IEEE. Personal use of this material is permitted. Permission from IEEE must be obtained for all other uses, in any current or future media, including reprinting/republishing this material for advertising or promotional purposes, creating new collective works, for resale or redistribution to servers or lists, or reuse of any copyrighted component of this work in other works. 


\title{
A Gaussian Mixture PHD Filter for Jump Markov System Models
}

\author{
Ahmed Pasha*, Ba-Ngu Vo ${ }^{\dagger}$, Hoang Duong Tuan ${ }^{\ddagger}$, Wing-Kin Ma ${ }^{\S}$
}

\begin{abstract}
The probability hypothesis density (PHD) filter is an attractive approach to tracking an unknown and time-varying number of targets in the presence of data association uncertainty, clutter, noise, and detection uncertainty. The PHD filter admits a closed form solution for a linear Gaussian multi-target model. However, this model is not general enough to accommodate maneuvering targets that switch between several models. In this paper, we generalize the notion of linear jump Markov systems to the multiple target case to accommodate births, deaths and switching dynamics. We then derive a closed form solution to the PHD recursion for the proposed linear Gaussian jump Markov multi-target model. Based on this an efficient method for tracking multiple maneuvering targets that switch between a set of linear Gaussian models is developed. An analytic implementation of the PHD filter using statistical linear regression technique is also proposed for targets that switch between a set of nonlinear models. We demonstrate through simulations that the proposed PHD filters are effective in tracking multiple maneuvering targets.
\end{abstract}

Index terms - Multi-target tracking, optimal filtering, point processes, random sets, intensity function, jump Markov models.

SP-EDICS: SSP-a Filtering, SSP-d Tracking algorithms, SSP-u Applications of statistical signal processing techniques

\footnotetext{
This work is supported in part by the discovery grant DP0556174 awarded by the Australian Research Council.

* Corresponding author. Address: School of Electrical Engineering and Telecommunications, The University of New South Wales, Sydney, NSW 2052, Australia. E-mail: s.pasha@student.unsw.edu.au～Tel.: +61 293855701

$\dagger$ Address: Department of Electrical and Electronic Engineering, The University of Melbourne, Parkville, Victoria 3010, Australia. E-mail: bv@ee.unimelb.edu.au Tel.: +61383446693 Fax: +61383446678

$\ddagger$ Address: School of Electrical Engineering and Telecommunications, The University of New South Wales, Sydney, NSW 2052, Australia. E-mail: h.d.tuan@unsw.edu.au Tel.: +61293855375 Fax: +61 293855993

$\S$ Address: Institute of Communications Engineering and Department of Electrical Engineering, National Tsing Hua University, 101, Section 2 Kuang Fu Road, Hsinchu,Taiwan 30013. E-mail: wkma@ieee.org Tel.: +88635715131 ext $34143 \quad$ Fax: +88635751787
} 


\section{INTRODUCTION}

While a non-maneuvering target motion can be described by a fixed model, a combination of motion models that characterise different maneuvers may be needed to describe the motion of a maneuvering target. Tracking a maneuvering target in clutter is a challenging problem and is the subject of numerous works [1]-[4]. In the multi-target setting, the number of targets changes due to targets appearing, disappearing, and it is not known which target generated which measurement. Tracking multiple maneuvering targets involves jointly estimating the number of targets and their states at each time step in the presence of noise, clutter, uncertainties in target maneuvers, data association and detection. As such, this problem is extremely challenging in both theory and implementation.

The jump Markov system (JMS) or multiple models approach has proven to be an effective tool for single maneuvering target tracking [2], [5]. In this approach, the target can switch between a set of models in a Markovian fashion. The JMS approach can also be combined with traditional data association techniques such as joint probabilistic data association (JPDA) [6]-[9] or multiple hypothesis tracking (MHT) [10], [11] to track multiple maneuvering targets. However, these data association-based approaches are computationally intensive in general and heuristic techniques are used to reduce the computational load.

Mahler's Probability Hypothesis Density (PHD) filter [12], [13] is a multi-target filter that circumvents the combinatorial computations due to data association while accommodating detection uncertainty, Poisson false alarms, target motion and time-varying number of targets. The generic sequential Monte Carlo implementation of the PHD filter [14], [15] can, in principle, accommodate any Markovian target dynamics including jump Markov systems. However, the drawbacks of the particle approach are the large number of particles, and the unreliability of clustering techniques for extracting state estimates [15], [16]. These problems are alleviated in the Gaussian mixture PHD filter implementation, which is developed from a closed form solution to the PHD recursion for linear Gaussian multi-target models [16], [17]. This approach is efficient and is capable of handling certain types non-linear models [16] but is not general enough to accommodate JMS models. At present there is no tractable analytical techniques for tracking multiple targets with JMS dynamics.

In this paper, we generalize the notion of linear jump Markov systems to the multiple target case to accommodate births, deaths and switching dynamics. We then derive a closed form solution to the PHD recursion for this so-called linear Gaussian jump Markov multi-target model. This solution generalizes the result in [16], [17] to a broader class of practical models. Based on this closed form solution, an efficient 
method is developed for tracking multiple maneuvering targets that switch between multiple models. Our approach can handle problems that are deemed intractable using traditional tracking techniques. Comparison with the classical IMMJPDA filter showed that the proposed approach is computationally much more efficient while exhibiting similar tracking performance, despite the fact that the IMMJPDA filter uses exact knowledge of the fixed number of targets. In addition, we extend our approach to nonlinear jump Markov multi-target models by combining our closed form solution and the unscented transform [18]. The proposed multi-target filters sidestep the data association problem and do not require clustering for extracting state estimates. Simulation results are presented to demonstrate the capability of the proposed method.

The paper is structured as follows: Section II presents some background on JMS for modelling a maneuvering target and the PHD filter. In section III we describe the JMS multi-target model for the PHD filter and give the main result of this paper, a closed-form solution to the PHD recursion for linear JMS and demonstrate the capability of the proposed algorithm through simulations. In Section IV we discuss the approximate solution to the PHD recursion for nonlinear JMS. This is followed by concluding remarks in Section V.

\section{Problem Formulation}

We review JMS and in particular the class of linear JMS for modeling maneuvering targets in Section II-A. Using the random finite set (RFS) representations for multi-target states and sensor measurements, our problem is posed as a Bayesian filtering problem in Section II-B. Section II-C describes the PHD filter.

\section{A. Jump Markov System (JMS)}

A jump Markov system (JMS) can be described by a set of parameterised state space models whose underlying parameters evolve with time according to a finite state Markov chain. Such a system finds a range of applications in signal processing and provides a natural means to model a maneuvering target whose behavior cannot be characterised at all times by a single model [3]-[5].

Let $\xi_{k} \in \mathbb{R}^{n}$ and $z_{k} \in \mathbb{R}^{m}$ denote the kinematic state (e.g. target coordinates and velocity) and observation, respectively, at time $k$. Suppose that $r_{k} \in \mathcal{M}$ is the label of the model in effect at time $k$, where $\mathcal{M}$ denotes the (discrete) set of all model labels (also called modes). Then, the state evolution and 
measurement are described by the transition density and measurement likelihood:

$$
\begin{aligned}
& \tilde{f}_{k \mid k-1}\left(\xi_{k} \mid \xi_{k-1}, r_{k}\right), \\
& g_{k}\left(z_{k} \mid \xi_{k}, r_{k}\right) .
\end{aligned}
$$

In addition, the modes follow a discrete Markov chain with transition probability $t_{k \mid k-1}\left(r_{k} \mid r_{k-1}\right)$ and the transition of the augmented state vector $x_{k}=\left[\xi_{k}^{T}, r_{k}\right]^{T} \in \mathcal{X}=\mathbb{R}^{n} \times \mathcal{M}$ is governed by

$$
f_{k \mid k-1}\left(x_{k} \mid x_{k-1}\right)=\tilde{f}_{k \mid k-1}\left(\xi_{k} \mid \xi_{k-1}, r_{k}\right) t_{k \mid k-1}\left(r_{k} \mid r_{k-1}\right)
$$

A linear Gaussian JMS (LGJMS) is a JMS with linear Gaussian models, i.e. conditioned on mode $r_{k}$ the state transition density and observation likelihood are given by

$$
\begin{aligned}
\tilde{f}_{k \mid k-1}\left(\xi_{k} \mid \xi_{k-1}, r_{k}\right) & =\mathcal{N}\left(\xi_{k} ; F_{k-1}\left(r_{k}\right) \xi_{k-1}, Q_{k}\left(r_{k}\right)\right), \\
g_{k}\left(z_{k} \mid \xi_{k}, r_{k}\right) & =\mathcal{N}\left(z_{k} ; H_{k}\left(r_{k}\right) \xi_{k}, R_{k}\left(r_{k}\right)\right)
\end{aligned}
$$

where $\mathcal{N}(\cdot ; m, Q)$ denotes a Gaussian density with mean $m$ and covariance $Q, F_{k-1}\left(r_{k}\right)$ and $H_{k}\left(r_{k}\right)$ denote the transition and observation matrices of model $r_{k} . Q_{k}\left(r_{k}\right)$ and $R_{k}\left(r_{k}\right)$ denote covariance matrices of the process noise and measurement noise.

Tracking a maneuvering target amounts to estimating the kinematic state $\xi_{k}$ or augmented state $x_{k}$ at time $k$, from the sequence of observations $z_{1: k}=\left(z_{1}, \ldots, z_{k}\right)$. The JMS (or multiple models) approach has been shown to be highly effective for maneuvering target tracking [2], [5].

\section{B. Random Finite Sets in Multi-target Tracking}

In a multi-target scenario, suppose that $x_{k, 1}, \ldots, x_{k, N(k)} \in \mathcal{X}$ are the augmented states at time $k$, where $N(k)$ denotes the number of targets. At the next time step, some of these targets may die, new targets may appear and the surviving targets evolve to their new states. At the sensor, $M(k)$ measurements $z_{k, 1}, \ldots, z_{k, M(k)} \in \mathbb{R}^{m}$ are received at time $k$, some of which are due to targets while the rest are clutter. Note that only some of the existing targets are detected by the sensor, and that the corresponding measurements are indistinguishable from clutter. Hence, the orders in which the states, and the measurements are listed bear no significance. Jointly estimating the time-varying number of states and the values of the states is a fundamentally difficult problem because in addition to the target maneuvers, the number of targets and the number of measurements both vary randomly in time and it is not known which target generated which measurement. 
Mahler's finite set statistics (FISST) [12], [13], [19] approach provides an elegant Bayesian formulation of the multi-target filtering problem by treating the finite sets of targets and observations, at time $k$, as the multi-target state and multi-target observation, respectively [12]

$$
\begin{aligned}
X_{k} & =\left\{x_{k, 1}, \ldots, x_{k, N(k)}\right\} \subset \mathcal{X}, \\
Z_{k} & =\left\{z_{k, 1}, \ldots, z_{k, M(k)}\right\} \subset \mathbb{R}^{m} .
\end{aligned}
$$

To model uncertainty in multi-target states and observations, we appeal to the notion of a random finite set (RFS). An RFS on a state space $\mathcal{X}$ is simply a random variable taking values in the finite subsets of $\mathcal{X}$ [20]. The intensity of an RFS on $\mathcal{X}$ is a non-negative function $v$ on $\mathcal{X}$ such that $v(x)$ is the instantaneous expected number of targets per unit volume at $x$. An RFS is Poisson if its cardinality distribution is Poisson with mean $N=\int v(x) d x$ and given a cardinality the elements of $X$ are i.i.d. according to $v / N$. We refer the reader to [15], [16] for overviews on FISST and [12], [13], [19] for comprehensive treatments.

Along the same vein as the single-target filtering problem, a multi-target transition density can be constructed from the RFS model for the time evolution of the multi-target state, which incorporates target motion, spontaneous births, spawnings (off existing targets) and deaths. Similarly, a multi-target likelihood can be constructed from the RFS measurement model, which accounts for detection uncertainty and clutter. The posterior distribution of the RFS of targets can be propagated in time by the multi-target Bayes recursion [12], [13], [15]. However, this recursion involves multiple integrals on the space of finite subsets of $\mathcal{X}$. In addition, the multi-target densities are combinatorial in nature. Hence, the multi-target Bayes filter is computationally intractable in general. Sequential Monte Carlo implementations can be found in [14], [15], [21]-[23], although these methods are still computationally intensive, especially when the number of targets is large.

\section{The Probability Hypothesis Density Filter}

An intelligent approximation to the multi-target Bayes filter, known as the Probability Hypothesis Density (PHD) filter, and which avoids any data association computations, has been proposed in [12]. The PHD filter propagates the posterior intensity of the RFS of targets in time, based on the following assumptions:

A. 1 Targets evolve in time and generate measurements independently of one another.

\section{A. 2 The clutter RFS is Poisson and is independent of the measurements.}




\section{A. 3 The predicted multi-target RFS is Poisson.}

Assumptions A.1 and A.2 are quite common in many multi-target tracking algorithms [1], [24]. The additional assumption A.3 is a reasonable approximation in applications where interactions between targets are negligible [12].

The PHD propagation is a recursion consisting of a prediction step and a data update step. Let $v_{k \mid k-1}$ and $v_{k}$ denote the predicted intensity and posterior intensity at time $k$, respectively. Then the $P H D$ prediction is given by

$$
v_{k \mid k-1}(x)=\int\left[p_{S, k \mid k-1}\left(x^{\prime}\right) f_{k \mid k-1}\left(x \mid x^{\prime}\right)+\beta_{k \mid k-1}\left(x \mid x^{\prime}\right)\right] v_{k-1}\left(x^{\prime}\right) d x^{\prime}+\gamma_{k}(x),
$$

where it is understood that an integral with respect to a discrete variable means a sum, and $f_{k \mid k-1}\left(\cdot \mid x^{\prime}\right)=$ probability density of a target at time $k$, given that its previous state is $x^{\prime}$, $p_{S, k \mid k-1}\left(x^{\prime}\right)=$ probability that a target still exists at time $k$ given that its previous state is $x^{\prime}$, $\beta_{k \mid k-1}\left(\cdot \mid x^{\prime}\right)=$ intensity of the RFS of targets spawned at time $k$ by a target with previous state $x^{\prime}$, $\gamma_{k}(\cdot) \quad=$ intensity of the birth RFS at time $k$.

On arrival of a new multi-target measurement, the posterior intensity $v_{k}$ is computed from the predicted intensity $v_{k \mid k-1}$ via the PHD update:

$$
v_{k}(x)=\left[1-p_{D, k}(x)+p_{D, k}(x) \sum_{z \in Z_{k}} \frac{g_{k}(z \mid x)}{\kappa_{k}(z)+\int p_{D, k}(x) g_{k}(z \mid x) v_{k \mid k-1}(x) d x}\right] v_{k \mid k-1}(x),
$$

where

$$
\begin{array}{ll}
Z_{k} & =\text { multi-target measurement at time } k, \\
g_{k}(\cdot \mid x) & =\text { single-target measurement likelihood at time } k, \\
p_{D, k}(x) & =\text { probability of detection given a state } x \text { at time } k, \\
\kappa_{k}(\cdot) & =\text { intensity of clutter RFS at time } k .
\end{array}
$$

The PHD recursion is generally intractable due to the 'curse of dimensionality' in numerical integration. A generic sequential Monte Carlo (SMC) implementation was proposed in [14], [15] with relevant convergence results (see also [25], [26] for more detailed asymptotic studies). This so-called particle-PHD filter can accommodate targets with JMS dynamics, and has been used to track multiple maneuvering targets in [27], [28]. However, the drawbacks of the particle approach are the large number of particles, and the unreliability of clustering techniques for extracting state estimates [15], [16]. The recently proposed 
Gaussian mixture PHD filter [16], [17] does not suffer from these drawbacks but is not general enough to handle JMS dynamics. In the following sections, we derive a closed form solution to the PHD recursion for LGJMS dynamics and develop an efficient and reliable multi-target filter for tracking maneuvering targets.

\section{CLOSED FORM SOLUTION TO THE PHD RECURSION FOR LGJMS MULTI-TARGET MODEL}

This section presents a closed-form PHD solution that can accommodate targets that switch between linear Gaussian models. We describe the LGJMS multi-target model in III-A and derive the corresponding closed form PHD recursion in III-B. In III-C, we derive a general closed form solution to the PHD recursion in the hybrid state space $\mathcal{X}=\mathbb{R}^{n} \times \mathcal{M}$. Illustrations of the proposed multi-target tracking algorithm on simulated data are given in III-D.

For notational convenience, the symbol $\Theta$ is used to denote the ordered pair of mean and covariance $(m, P)$ of a Gaussian distribution, i.e

$$
\mathcal{N}(x ; \Theta)=\mathcal{N}(x ; m, P) .
$$

Given a linear Gaussian model $z=H x+v$, where $v$ is Gaussian noise with mean $d$ and covariance matrix $R$, we use the notation $\Omega$ to denote the ordered triplet of model parameters $(H, R, d)$, and

$$
\mathcal{L}(x, z ; \Omega)=\mathcal{N}(z ; H x+d, R)
$$

to denote the probability density at $z$. This notation is suggestive of the mapping of $x$ to $z$ via the linear model with parameter $\Omega$. Note that $\mathcal{N}(x ; m, P)=\mathcal{L}(m, x ;(I, P, 0))=\mathcal{L}(x, m ;(I, P, 0))$.

\section{A. Linear Gaussian Jump Markov System Multi-target Models}

This subsection presents the linear Gaussian JMS (LGJMS) multi-target model, which accommodates targets with switching linear dynamics. Campbell's theorem [29] is used in the modelling of target births and spawning.

In addition to assumptions A.1 - A.3, the LGJMS multi-target model comprises a LGJMS model for individual targets, kinematic-independent survival and detection probabilities, and models for target births and spawnings. Like the motion model, birth and spawning models are naturally described in terms of the kinematic state. However, while the distribution of the augmented state can be taken as the product of the mode distribution and the kinematic state distribution conditional on the mode, i.e. $p(\xi, r)=p(r) p(\xi \mid r)$, this line of reasoning does not extend to birth and spawning intensities. The intensity of the augmented 
state is not necessarily the product of the intensity of the mode and the intensity of the kinematic state conditioned on the mode.

To specify birth and spawning models for the kinematic state and mode that yield valid birth and spawning intensities in the augmented state, we appeal to a well-known result in point process theory, namely Campbell's theorem (for marked point processes) [29, pp. 106-108]. In particular, Campbell's theorem implies that the intensity of the point process on $\mathbb{R}^{n} \times \mathcal{M}$ formed by the Cartesian product of a point process on the kinematic state space $\mathbb{R}^{n}$, with intensity $\tilde{v}$, and a point process on the mode space $\mathcal{M}$, is given by

$$
v(\xi, r)=p(r \mid \xi) \tilde{v}(\xi)
$$

where $p(\cdot \mid \xi)$ is the mode distribution given that a point of the product point process has kinematic state $\xi$. Moreover, if the point process on $\mathbb{R}^{n}$ is Poisson, then the product point process on $\mathbb{R}^{n} \times \mathcal{M}$ is also Poisson [30].

1) Birth model 1: In the context of our multi-target birth model, the intensity of augmented state births at time $k$ is given by

$$
\gamma_{k}(\xi, r)=\pi_{k}(r \mid \xi) \tilde{\gamma}_{k}(\xi)
$$

where $\tilde{\gamma}_{k}$ is the intensity of kinematic state births at time $k$, and $\pi_{k}(\cdot \mid \xi)$ is the probability distribution of the modes for a given birth with kinematic state $\xi$ at time $k$. In line with the standard LGJMS assumption that the mode transition probability $t_{k \mid k-1}$ is not a function of the kinematic states, the LGJMS multi-target model also assumes that the mode distribution does not depend on the kinematic state, i.e. $\pi_{k}(r \mid \xi)=\pi_{k}(r)$. Moreover, it is also assumed that the intensity $\tilde{\gamma}_{k}$ of kinematic state births is a Gaussian mixture

$$
\tilde{\gamma}_{k}(\xi)=\sum_{i=1}^{J_{\gamma, k}} w_{\gamma, k}^{(i)} \mathcal{N}\left(\xi ; \Theta_{\gamma, k}^{(i)}\right),
$$

where $J_{\gamma, k}, w_{\gamma, k}^{(i)}, \Theta_{\gamma, k}^{(i)}=\left(m_{\gamma, k}^{(i)}, Q_{\gamma, k}^{(i)}\right), i=1,2, \ldots, J_{\gamma, k}$ are given model parameters. The mean $m_{\gamma, k}^{(i)}$ is a peak of the intensity $\tilde{\gamma}_{k}$ and has the highest local concentrations of expected number of births, and represents, for example, airbases or airports where targets are most likely to appear. The covariance matrix $P_{\gamma, k}^{(i)}$ determines the spread of $\tilde{\gamma}_{k}$ in the vicinity of the peak $m_{\gamma, k}^{(i)}$. The weight $w_{\gamma, k}^{(i)}$ gives the expected number target births originating from $m_{\gamma, k}^{(i)}$.

Similarly, the intensity of augmented states spawned, at time $k$, from a target with augmented state $\left[\xi^{\prime}, r^{\prime}\right]^{T}$, at time $k-1$, is given by

$$
\beta_{k \mid k-1}\left(\xi, r \mid \xi^{\prime}, r^{\prime}\right)=\pi_{k \mid k-1}\left(r \mid \xi, \xi^{\prime}, r^{\prime}\right) \tilde{\beta}_{k \mid k-1}\left(\xi \mid \xi^{\prime}, r^{\prime}\right)
$$


where $\tilde{\beta}_{k \mid k-1}\left(\cdot \mid \xi^{\prime}, r^{\prime}\right)$ is the intensity of kinematic states spawned at time $k$ from $\left[\xi^{\prime}, r^{\prime}\right]^{T}$, and $\pi_{k \mid k-1}\left(\cdot \mid \xi, \xi^{\prime}, r^{\prime}\right)$ is the probability distribution of the mode for a given kinematic state $\xi$, spawned at time $k$ from $\left[\xi^{\prime}, r^{\prime}\right]^{T}$. Consistent with standard LGJMS assumption, the LGJMS multi-target model assumes that the mode distribution of a spawned target does not depend on its kinematic state nor its parent's kinematic state, i.e. $\pi_{k \mid k-1}\left(r \mid \xi, \xi^{\prime}, r^{\prime}\right)=\pi_{k \mid k-1}\left(r \mid r^{\prime}\right)$, and that the intensity $\tilde{\beta}_{k \mid k-1}\left(\cdot \mid \xi^{\prime}, r^{\prime}\right)$ of spawned kinematic states is a Gaussian mixture

$$
\tilde{\beta}_{k \mid k-1}\left(\xi \mid \xi^{\prime}, r^{\prime}\right)=\sum_{j=1}^{J_{\beta, k}\left(r^{\prime}\right)} w_{\beta, k \mid k-1}^{(j)}\left(r^{\prime}\right) \mathcal{L}\left(\xi^{\prime}, \xi ; \Omega_{\beta, k \mid k-1}^{(j)}\left(r^{\prime}\right)\right),
$$

where $J_{\beta, k \mid k-1}\left(r^{\prime}\right), w_{\beta, k \mid k-1}^{(j)}\left(r^{\prime}\right), \Omega_{\beta, k \mid k-1}^{(j)}\left(r^{\prime}\right)=\left(F_{\beta, k-1}^{(j)}\left(r^{\prime}\right), Q_{\beta, k-1}^{(j)}\left(r^{\prime}\right), d_{\beta, k-1}^{(j)}\left(r^{\prime}\right)\right), j=1,2, \ldots$, $J_{\beta, k-1}\left(r^{\prime}\right)$ are given model parameters. A similar interpretation to $\tilde{\gamma}_{k}$ applies to the intensity $\tilde{\beta}_{k \mid k-1}$, except that the $j$ th peak, $F_{\beta, k-1}^{(j)}\left(r^{\prime}\right) \xi^{\prime}+d_{\beta, k-1}^{(j)}\left(r^{\prime}\right)$, is an affine function of $\xi^{\prime}$. Usually, a spawned target is modelled to be in the proximity of its parent.

2) Birth model 2: Alternatively, by interchanging the roles of the kinematic state space and mode space in (8), consistent models for births and spawnings can also be derived ${ }^{1}$. In this case, the intensity of augmented state births at time $k$ is given by

$$
\gamma_{k}(\xi, r)=\tilde{\gamma}(\xi \mid r) \pi_{k}(r)
$$

where $\pi_{k}$ is now the intensity of mode births and $\tilde{\gamma}_{k}(\cdot \mid r)$ is now the distribution of the birth kinematic state given mode $r$. Note that the intensity of mode births is not a function of kinematic state. It is assumed, in the LGJMS multi-target model, that the distribution $\tilde{\gamma}_{k}(\cdot \mid r)$ of kinematic state births is a Gaussian mixture

$$
\tilde{\gamma}_{k}(\xi \mid r)=\sum_{i=1}^{J_{\gamma, k}(r)} w_{\gamma, k}^{(i)}(r) \mathcal{N}\left(\xi ; \Theta_{\gamma, k}^{(i)}(r)\right),
$$

where $J_{\gamma, k}(r), w_{\gamma, k}^{(i)}(r), \Theta_{\gamma, k}^{(i)}(r)=\left(m_{\gamma, k}^{(i)}(r), Q_{\gamma, k}^{(i)}(r)\right), i=1,2, \ldots, J_{\gamma, k}(r)$ are given model parameters that depend on the mode $r$. Similarly, the intensity of augmented states spawned, at time $k$, spawned from $\left[\xi^{\prime}, r^{\prime}\right]^{T}$ is

$$
\beta_{k \mid k-1}\left(\xi, r \mid \xi^{\prime}, r^{\prime}\right)=\tilde{\beta}_{k \mid k-1}\left(\xi \mid r, \xi^{\prime}, r^{\prime}\right) \pi_{k \mid k-1}\left(r \mid \xi^{\prime}, r^{\prime}\right),
$$

where $\pi_{k \mid k-1}\left(\cdot \mid \xi^{\prime}, r^{\prime}\right)$ is now the intensity of mode spawnings and $\tilde{\beta}_{k \mid k-1}\left(\cdot \mid r, \xi^{\prime}, r^{\prime}\right)$ is now the distribution of spawned kinematic state given mode $r$. The LGJMS multi-target model assumes that the intensity of

\footnotetext{
${ }^{1}$ One technicality is that we need to restrict the kinematic state space to a compact subset of $R^{n}$. This technicality does not pose any problem in practice since the targets occupy a bounded region of space.
} 
spawned modes does not depend on the kinematic state of its parent, i.e. $\pi_{k \mid k-1}\left(r \mid \xi^{\prime}, r^{\prime}\right)=\pi_{k \mid k-1}\left(r \mid r^{\prime}\right)$, and that the distribution $\tilde{\beta}_{k \mid k-1}\left(\cdot \mid r, \xi^{\prime}, r^{\prime}\right)$ of the spawned kinematic state is a Gaussian mixture

$$
\tilde{\beta}_{k \mid k-1}\left(\xi \mid r, \xi^{\prime}, r^{\prime}\right)=\sum_{j=1}^{J_{\beta, k}\left(r, r^{\prime}\right)} w_{\beta, k \mid k-1}^{(j)}\left(r, r^{\prime}\right) \mathcal{L}\left(\xi^{\prime}, \xi ; \Omega_{\beta, k \mid k-1}^{(j)}\left(r, r^{\prime}\right)\right),
$$

where $J_{\beta, k \mid k-1}\left(r, r^{\prime}\right), w_{\beta, k \mid k-1}^{(j)}\left(r, r^{\prime}\right), \Omega_{\beta, k \mid k-1}^{(j)}\left(r, r^{\prime}\right)=\left(F_{\beta, k-1}^{(j)}\left(r, r^{\prime}\right), Q_{\beta, k-1}^{(j)}\left(r, r^{\prime}\right), d_{\beta, k-1}^{(j)}\left(r, r^{\prime}\right)\right), j=1$, $2, \ldots, J_{\beta, k \mid k-1}\left(r, r^{\prime}\right)$ are given model parameters that depend on the current mode $r$ and the parent's previous mode $r^{\prime}$.

From a modelling and application point of view, models 1 and 2 are different. However, from an algorithmic or computational viewpoint, the first model can be treated as a special case of the second model with the distribution of the birth kinematic state being independent of mode $r$, i.e., $\tilde{\gamma}_{k}(\xi \mid r)=\tilde{\gamma}_{k}(\xi)$.

Summarizing, in addition to assumptions A.1 - A.3, the linear Gaussian JMS (LGJMS) multi-target model, assumes:

A. 4 Each target follows a LGJMS model, i.e. the dynamic and measurement models for the augmented state have the form:

$$
\begin{aligned}
f_{k \mid k-1}\left(\xi, r \mid \xi^{\prime}, r^{\prime}\right) & =\mathcal{L}\left(\xi^{\prime}, \xi ; \Omega_{f, k \mid k-1}(r)\right) t_{k \mid k-1}\left(r \mid r^{\prime}\right), \\
g_{k}(z \mid \xi, r) & =\mathcal{L}\left(\xi, z ; \Omega_{g, k}(r)\right),
\end{aligned}
$$

where $\Omega_{f, k \mid k-1}(r)=\left(F_{f, k-1}(r), Q_{f, k}(r), 0\right)$ is the parameter of the linear target dynamics model conditioned on mode $r, \Omega_{g, k}(r)=\left(H_{k}(r), R_{k}(r), 0\right)$ is the parameters of the linear observation model conditioned on mode $r$, and $t_{k \mid k-1}\left(r \mid r^{\prime}\right)$ is the mode transition probability. In particular, conditional on mode $r, F_{f, k-1}(r)$ is the state transition matrix, $Q_{f, k}(r)$ is the process noise covariance matrix, $H_{k}(r)$ is the measurement matrix and $R_{k}(r)$ is the measurement noise covariance matrix.

A. 5 The probabilities of target survival and target detection are independent of the kinematic state:

$$
\begin{aligned}
p_{S, k \mid k-1}\left(\xi^{\prime}, r^{\prime}\right) & =p_{S, k \mid k-1}\left(r^{\prime}\right) \\
p_{D, k}(\xi, r) & =p_{D, k}(r) .
\end{aligned}
$$

Assumptions A.4 and A.5 follow from those commonly used in maneuvering target tracking algorithms (see for example [1], [24], [31]), 
A. 6 The intensities of the birth and spawn RFSs can be expressed as Gaussian mixtures of the form:

$$
\begin{aligned}
\gamma_{k}(\xi, r) & =\pi_{k}(r) \sum_{i=1}^{J_{\gamma, k}(r)} w_{\gamma, k}^{(i)}(r) \mathcal{N}\left(\xi ; \Theta_{\gamma, k}^{(i)}(r)\right), \\
\beta_{k \mid k-1}\left(\xi, r \mid \xi^{\prime}, r^{\prime}\right) & =\pi_{k \mid k-1}\left(r \mid r^{\prime}\right) \sum_{j=1}^{J_{\beta, k}\left(r, r^{\prime}\right)} w_{\beta, k \mid k-1}^{(j)}\left(r, r^{\prime}\right) \mathcal{L}\left(\xi^{\prime}, \xi ; \Omega_{\beta, k \mid k-1}^{(j)}\left(r, r^{\prime}\right)\right),
\end{aligned}
$$

where $J_{\gamma, k}(r), w_{\gamma, k}^{(i)}(r), \Theta_{\gamma, k}^{(i)}(r)=\left(m_{\gamma, k}^{(i)}(r), Q_{\gamma, k}^{(i)}(r)\right), i=1,2, \ldots, J_{\gamma, k}(r)$ are given parameters of the (Gaussian mixture) density of the kinematic state of a new born target with mode $r$ at time $k$, and $\pi_{k}(\cdot)$ is the intensity of mode births at time $k$. Similarly, $J_{\beta, k}\left(r, r^{\prime}\right), w_{\beta, k \mid k-1}^{(j)}\left(r, r^{\prime}\right), \Omega_{\beta, k \mid k-1}^{(j)}\left(r, r^{\prime}\right)=$ $\left(F_{\beta, k-1}^{(j)}\left(r, r^{\prime}\right), Q_{\beta, k-1}^{(j)}\left(r, r^{\prime}\right), d_{\beta, k-1}^{(j)}\left(r, r^{\prime}\right)\right), \quad j=1,2, \ldots, J_{\beta, k-1}\left(r, r^{\prime}\right)$ are given parameters of the (Gaussian mixture) density of the kinematic state of a target with mode $r$, spawned at time $k$ from a target with augmented state $\left[\xi^{\prime}, r^{\prime}\right]^{T}$ at time $k-1$, and $\pi_{k \mid k-1}\left(\cdot \mid r^{\prime}\right)$ is the intensity of modes spawned at time $k$ from a target with mode $r^{\prime}$ at time $k-1$.

The LGJMS multi-target model is more general than those in standard multi-target tracking algorithms. While most existing algorithms do not account for births or spawnings, the proposed multi-target model incorporates both. Models for births and spawnings for a given mode $r$ accommodate different intensities of mode births and modes spawned respectively when births and spawnings are likely to vary between different modes. Similarly, the proposed model incorporates models for target death (survival) and target detection for a given mode $r$. Moreover, traditional multi-target filtering techniques are computationally intractable for a model of such generality. We will see later that using a random finite set approach [12], this model is amenable to computationally efficient multi-target filtering techniques.

\section{B. Closed form PHD recursion for LGJMS multi-target model}

To derive the closed form PHD recursion for the LGJMS multi-target model, we require Lemmas 1 and 2 in [16], [17], which are stated using the new notation as follows:

Lemma 1: If $\Omega=(H, R, d)$ and $\Theta=(m, P)$, then

$$
\int \mathcal{L}(x, z ; \Omega) \mathcal{N}(x ; \Theta) d x=\mathcal{N}(z ; \Pi(\Omega, \Theta))
$$

where

$$
\Pi(\Omega, \Theta)=\left(H m+d, R+H P H^{T}\right)
$$

Lemma 2: If $\Omega=(H, R, d)$ and $\Theta=(m, P)$, then

$$
\mathcal{L}(x, z ; \Omega) \mathcal{N}(x ; \Theta)=\mathcal{N}(z ; \Pi(\Omega, \Theta)) \mathcal{N}(x ; \Psi(z, \Omega, \Theta))
$$


where

$$
\begin{aligned}
\Psi(z, \Omega, \Theta) & =(\tilde{m}(z-d), \tilde{P}) \\
\tilde{m}(z-d) & =m+K(z-d-H m) \\
\tilde{P} & =(I-K H) P \\
K & =P H^{T}\left(H P H^{T}+R\right)^{-1}
\end{aligned}
$$

Proposition 1: For a LGJMS multi-target model, if the posterior intensity $v_{k-1}$ at time $k-1$ has the form

$$
v_{k-1}\left(\xi^{\prime}, r^{\prime}\right)=\sum_{i=1}^{J_{k-1}\left(r^{\prime}\right)} w_{k-1}^{(i)}\left(r^{\prime}\right) \mathcal{N}\left(\xi^{\prime} ; \Theta_{k-1}^{(i)}\left(r^{\prime}\right)\right) .
$$

Then the predicted intensity $v_{k \mid k-1}$ is given by

$$
v_{k \mid k-1}(\xi, r)=\gamma_{k}(\xi, r)+v_{f, k \mid k-1}(\xi, r)+v_{\beta, k \mid k-1}(\xi, r),
$$

where

$$
\begin{aligned}
v_{\beta, k \mid k-1}(\xi, r) & =\sum_{r^{\prime}} \sum_{i=1}^{J_{k-1}\left(r^{\prime}\right)} \sum_{j=1}^{J_{\beta, k \mid k-1}\left(r, r^{\prime}\right)} w_{\beta, k \mid k-1}^{(i, j)}\left(r, r^{\prime}\right) \mathcal{N}\left(\xi ; \Theta_{\beta, k \mid k-1}^{(i, j)}\left(r, r^{\prime}\right)\right), \\
w_{\beta, k \mid k-1}^{(i, j)}\left(r, r^{\prime}\right) & =\pi_{k \mid k-1}\left(r \mid r^{\prime}\right) w_{\beta, k \mid k-1}^{(j)}\left(r, r^{\prime}\right) w_{k-1}^{(i)}\left(r^{\prime}\right), \\
\Theta_{\beta, k \mid k-1}^{(i, j)}\left(r, r^{\prime}\right) & =\Pi\left(\Omega_{\beta, k \mid k-1}^{(j)}\left(r, r^{\prime}\right), \Theta_{k-1}^{(i)}\left(r^{\prime}\right)\right), \\
v_{f, k \mid k-1}(\xi, r) & =\sum_{r^{\prime}} \sum_{i=1}^{J_{k-1}\left(r^{\prime}\right)} w_{f, k \mid k-1}^{(i)}\left(r, r^{\prime}\right) \mathcal{N}\left(\xi ; \Theta_{f, k \mid k-1}^{(i)}\left(r, r^{\prime}\right)\right), \\
w_{f, k \mid k-1}^{(i)}\left(r, r^{\prime}\right) & =p_{S, k \mid k-1}\left(r^{\prime}\right) t_{k \mid k-1}\left(r \mid r^{\prime}\right) w_{k-1}^{(i)}\left(r^{\prime}\right), \\
\Theta_{f, k \mid k-1}^{(i)}\left(r, r^{\prime}\right) & =\Pi\left(\Omega_{f, k \mid k-1}(r), \Theta_{k-1}^{(i)}\left(r^{\prime}\right)\right) .
\end{aligned}
$$

Proof: From (8), the predicted intensity consists of three terms $\gamma_{k}$ (already given in the multitarget model), $v_{\beta, k \mid k-1}$ and $v_{f, k \mid k-1}$, due to births, spawnings and motion, respectively. For $v_{\beta, k \mid k-1}$, substituting (22), (29) into $\int \beta_{k \mid k-1}\left(x \mid x^{\prime}\right) v_{k-1}\left(x^{\prime}\right) d x^{\prime}$, exchanging the order of sums and integral, and applying Lemma 1 to individual terms yields (31). For $v_{f, k \mid k-1}$ we substitute (17) and (29) into $\int f_{k \mid k-1}\left(x \mid x^{\prime}\right) v_{k-1}\left(x^{\prime}\right) d x^{\prime}$, exchange the order of sums and integral, and apply Lemma 1 to individual terms to obtain (34).

Corollary 1: Under the premises of Proposition 1, the expected number of predicted targets is

$$
\hat{N}_{k \mid k-1}=\hat{N}_{\gamma, k}+\hat{N}_{f, k \mid k-1}+\hat{N}_{\beta, k \mid k-1},
$$


where

$$
\begin{aligned}
\hat{N}_{\gamma, k} & =\sum_{r} \sum_{i=1}^{J_{\gamma, k}(r)} \pi_{k}(r) w_{\gamma, k}^{(i)}(r), \\
\hat{N}_{\beta, k \mid k-1} & =\sum_{r} \sum_{r^{\prime}} \sum_{i=1}^{J_{k-1}\left(r^{\prime}\right)} \sum_{j=1}^{J_{\beta, k \mid k-1}\left(r, r^{\prime}\right)} \pi_{k \mid k-1}\left(r \mid r^{\prime}\right) w_{\beta, k \mid k-1}^{(j)}\left(r, r^{\prime}\right) w_{k-1}^{(i)}\left(r^{\prime}\right), \\
\hat{N}_{f, k \mid k-1} & =\sum_{r} \sum_{r^{\prime}} \sum_{i=1}^{J_{k-1}\left(r^{\prime}\right)} p_{S, k \mid k-1}\left(r^{\prime}\right) t_{k \mid k-1}\left(r \mid r^{\prime}\right) w_{k-1}^{(i)}\left(r^{\prime}\right),
\end{aligned}
$$

Proposition 2: For a LGJMS multi-target model, if the predicted intensity $v_{k \mid k-1}$ has the form

$$
v_{k \mid k-1}(\xi, r)=\sum_{i=1}^{J_{k \mid k-1}(r)} w_{k \mid k-1}^{(i)}(r) \mathcal{N}\left(\xi ; \Theta_{k \mid k-1}^{(i)}(r)\right) .
$$

Then the posterior intensity $v_{k}$ is given by

$$
v_{k}(\xi, r)=\left(1-p_{D, k}(r)\right) v_{k \mid k-1}(\xi, r)+\sum_{z \in Z_{k}} v_{g, k}(\xi, r ; z)
$$

where

$$
\begin{aligned}
v_{g, k}(\xi, r ; z) & =\sum_{i=1}^{J_{k \mid k-1}(r)} w_{g, k}^{(i)}(r ; z) \mathcal{N}\left(\xi ; \Theta_{g, k}^{(i)}(r ; z)\right), \\
w_{g, k}^{(i)}(r ; z) & =\frac{p_{D, k}(r) w_{k \mid k-1}^{(i)}(r) q_{g, k}^{(i)}(r ; z)}{\kappa_{k}(z)+\sum_{r} p_{D, k}(r) \sum_{i=1}^{J_{k \mid k-1}(r)} w_{k \mid k-1}^{(i)}(r) q_{g, k}^{(i)}(r ; z)}, \\
q_{g, k}^{(i)}(r ; z) & =\mathcal{N}\left(z ; \Pi\left(\Omega_{g, k}(r), \Theta_{k \mid k-1}^{(i)}(r)\right)\right), \\
\Theta_{g, k}^{(i)}(r ; z) & =\Psi\left(z, \Omega_{g, k}(r), \Theta_{k \mid k-1}^{(i)}(r)\right) .
\end{aligned}
$$

Proof: From (9), the updated intensity consists of three components. The first is the predicted intensity $v_{k \mid k-1}$ (given), the second is the product $p_{D, k} v_{k \mid k-1}$ denoted as $v_{D, k}$, and the third is the $\operatorname{sum} \sum_{z \in Z_{k}} v_{g, k}(x ; z)$, where

$$
v_{g, k}(x ; z)=\frac{g_{k}(z \mid x) v_{D, k}(x)}{\kappa_{k}(z)+\int g_{k}(z \mid x) v_{D, k}(x) d x} .
$$

For $v_{g, k}$, first substitute (18), (41) into the numerator of (47) and apply Lemma 2 to yield a sum of weighted Gaussians. Second, applying Lemma 1 to the integral in the denominator of (47) gives the (double) sum in the denominator of (44). Combining the results for the numerator and denominator of (47) gives (43). 
Corollary 2: Under the premises of Proposition 2, the expected number of target is

$$
\hat{N}_{k}=\sum_{r}\left[1-p_{D, k}(r)\right] \sum_{i=1}^{J_{k \mid k-1}(r)} w_{k \mid k-1}^{(i)}(r)+\sum_{z \in Z_{k}} \sum_{r} \sum_{i=1}^{J_{k \mid k-1}(r)} w_{g, k}^{(i)}(r ; z) .
$$

Propositions 1 and 2 show how the intensities $v_{k \mid k-1}$ and $v_{k}$ are analytically propagated in time under linear Gaussian assumption on the JMS multi-target model. The recursions for the means and covariances of $v_{f, k \mid k-1}$ and $v_{\beta, k \mid k-1}$ are the Kalman prediction and the recursive computations of the means and covariances of $v_{D, k}$ are the Kalman update. The PHD filter has a complexity of $\mathcal{O}\left(J_{k-1}\left|Z_{k}\right|\right)$ where $J_{k-1}$ is the number of Gaussian components representing $v_{k-1}$ for a fixed model $r^{\prime}$ at time $k-1$ and $\left|Z_{k}\right|$ denotes the number measurements at time $k$.

These propositions also indicate that the number of components of the predicted and posterior intensity increases with time, which can be a problem in implementation. However, this problem can be effectively handled by applying some simple pruning procedures [16], [17].

Given the posterior intensity $v_{k}$ at time $k$

$$
v_{k}(\xi, r)=\sum_{i=1}^{J_{k}(r)} w_{k}^{(i)}(r) \mathcal{N}\left(\xi ; \Theta_{k}^{(i)}(r)\right)
$$

the peaks of the intensity are points of highest local concentration of the expected number of targets. In order to extract the state of the targets from the posterior intensity at time $k$, an estimate of the number of targets $\hat{N}_{k}$ is needed. This number is simply $\sum_{i=1}^{J_{k}(r)} w_{k}^{(i)}(r)$ rounded to the nearest integer. The estimate of the multi-target state is the set of $\hat{N}_{k}$ ordered pairs of means and modes $\left(m_{k}^{(i)}(r), r\right)$ with the largest weights $w_{k}^{(i)}(r), r \in \mathcal{M}, i=1, \ldots, J_{k}(r)$.

\section{General solution to the PHD recursion}

Apart from the LG and LGJMS multi-target models, the PHD recursion also admits closed form solutions under more general settings. In this section we derive a general analytic solution to the PHD recursion in the hybrid state space $\mathcal{X}=\mathbb{R}^{n} \times \mathcal{M}$. Readers who are interested in the simulation results of the above developed PHD filter may proceed directly to Section III-D. 
Proposition 3: Given a multi-target transition model with

$$
\begin{aligned}
p_{S, k \mid k-1}\left(\xi^{\prime}, r^{\prime}\right) & =w_{S, k \mid k-1}^{(0)}\left(r^{\prime}\right)+\sum_{l=1}^{J_{S, k \mid k-1}\left(r^{\prime}\right)} w_{S, k \mid k-1}^{(l)}\left(r^{\prime}\right) \mathcal{L}\left(\xi^{\prime}, m_{S, k \mid k-1}^{(l)}\left(r^{\prime}\right) ; \Omega_{S, k \mid k-1}^{(l)}\left(r^{\prime}\right)\right), \\
f_{k \mid k-1}\left(\xi, r \mid \xi^{\prime}, r^{\prime}\right) & =\sum_{j=1}^{J_{f, k \mid k-1}\left(r, r^{\prime}\right)} w_{f, k \mid k-1}^{(j)}\left(r, r^{\prime}\right) \mathcal{L}\left(\xi^{\prime}, \xi ; \Omega_{f, k \mid k-1}^{(j)}\left(r, r^{\prime}\right)\right), \\
\beta_{k \mid k-1}\left(\xi, r \mid \xi^{\prime}, r^{\prime}\right) & =\sum_{j=1}^{J_{\beta, k \mid k-1}\left(r, r^{\prime}\right)} w_{\beta, k \mid k-1}^{(j)}\left(r, r^{\prime}\right) \mathcal{L}\left(\xi^{\prime}, \xi ; \Omega_{\beta, k \mid k-1}^{(j)}\left(r, r^{\prime}\right)\right) .
\end{aligned}
$$

If the posterior intensity $v_{k-1}$ at time $k-1$ has the form

$$
v_{k-1}\left(\xi^{\prime}, r^{\prime}\right)=\sum_{i=1}^{J_{k-1}\left(r^{\prime}\right)} w_{k-1}^{(i)}\left(r^{\prime}\right) \mathcal{N}\left(\xi^{\prime} ; \Theta_{k-1}^{(i)}\left(r^{\prime}\right)\right) .
$$

Then the predicted intensity $v_{k \mid k-1}$ is given by

$$
v_{k \mid k-1}(\xi, r)=\gamma_{k}(\xi, r)+v_{f, k \mid k-1}(\xi, r)+v_{\beta, k \mid k-1}(\xi, r),
$$

where

$$
\begin{aligned}
v_{\beta, k \mid k-1}(\xi, r) & =\sum_{r^{\prime}} \sum_{i=1}^{J_{k-1}\left(r^{\prime}\right)} \sum_{j=1}^{J_{\beta, k \mid k-1}\left(r, r^{\prime}\right)} w_{\beta, k \mid k-1}^{(i, j)}\left(r, r^{\prime}\right) \mathcal{N}\left(\xi ; \Theta_{\beta, k \mid k-1}^{(i, j)}\left(r, r^{\prime}\right)\right) \\
w_{\beta, k \mid k-1}^{(i, j)}\left(r, r^{\prime}\right) & =w_{\beta, k \mid k-1}^{(j)}\left(r, r^{\prime}\right) w_{k-1}^{(i)}\left(r^{\prime}\right), \\
\Theta_{\beta, k \mid k-1}^{(i, j)}\left(r, r^{\prime}\right) & =\Pi\left(\Omega_{\beta, k \mid k-1}^{(j)}\left(r, r^{\prime}\right), \Theta_{k-1}^{(i)}\left(r^{\prime}\right)\right), \\
v_{f, k \mid k-1}(\xi, r) & =\sum_{r^{\prime}} \sum_{i=1}^{J_{k-1}\left(r^{\prime}\right) J_{S, k \mid k-1}\left(r^{\prime}\right) J_{f, k \mid k-1}\left(r, r^{\prime}\right)} \sum_{l=0} \sum_{j=1}^{(i, j, l)} w_{f, k \mid k-1}\left(r, r^{\prime}\right) \mathcal{N}\left(\xi ; \Theta_{f, k \mid k-1}^{(i, j, l)}\left(r, r^{\prime}\right)\right) \\
w_{f, k \mid k-1}^{(i, j, l)}\left(r, r^{\prime}\right) & =w_{f, k \mid k-1}^{(j)}\left(r, r^{\prime}\right) w_{S, k \mid k-1}^{(l)}\left(r^{\prime}\right) w_{k-1}^{(i)}\left(r^{\prime}\right) q_{S, k \mid k-1}^{(i, l)}\left(r^{\prime}\right), \\
q_{S, k \mid k-1}^{(i, l)}\left(r^{\prime}\right) & =\mathcal{N}\left(m_{S, k \mid k-1}^{(l)}\left(r^{\prime}\right) ; \Pi\left(\Omega_{S, k \mid k-1}^{(l)}\left(r^{\prime}\right), \Theta_{k-1}^{(i)}\left(r^{\prime}\right)\right)\right), \quad q_{S, k \mid k-1}^{(i, 0)}\left(r^{\prime}\right)=1, \\
\Theta_{f, k \mid k-1}^{(i, j, l)}\left(r, r^{\prime}\right) & =\Pi\left(\Omega_{f, k \mid k-1}^{(j)}\left(r, r^{\prime}\right), \Theta_{S, k \mid k-1}^{(i, l)}\left(r^{\prime}\right)\right), \quad \Theta_{S, k \mid k-1}^{(i, 0)}\left(r^{\prime}\right)=\Theta_{k-1}^{(i)}\left(r^{\prime}\right) . \\
\Theta_{S, k \mid k-1}^{(i, l)}\left(r^{\prime}\right) & =\Psi\left(m_{S, k \mid k-1}^{(l)}\left(r^{\prime}\right), \Omega_{S, k \mid k-1}^{(l)}\left(r^{\prime}\right), \Theta_{k-1}^{(i)}\left(r^{\prime}\right)\right), \quad
\end{aligned}
$$

Proof: $v_{\beta, k \mid k-1}$ is obtained as before. For $v_{f, k \mid k-1}$ we first substitute (50), (53) into $p_{S, k \mid k-1}\left(x^{\prime}\right) v_{k-1}\left(x^{\prime}\right)$ and applying Lemma 2 to yield a (double) sum of weighted Gaussians. We then substitute the resulting Gaussian mixture and (51) into $\int p_{S, k \mid k-1}\left(x^{\prime}\right) f_{k \mid k-1}\left(x \mid x^{\prime}\right) v_{k-1}\left(x^{\prime}\right) d x^{\prime}$, exchange the order of sums and integral, and apply Lemma 1 to individual terms to obtain (58). 
Proposition 4: Given a multi-target measurement model with

$$
\begin{aligned}
p_{D, k}(\xi, r) & =w_{D, k}^{(0)}(r)+\sum_{l=1}^{J_{D, k}(r)} w_{D, k}^{(l)}(r) \mathcal{L}\left(\xi ; m_{D, k}^{(l)}(r), \Omega_{D, k}^{(l)}\left(r^{\prime}\right)\right), \\
g_{k}(z \mid \xi, r) & =\sum_{j=1}^{J_{g, k}(r)} w_{g, k}^{(j)}(r) \mathcal{M}\left(\xi ; z, \Omega_{g, k}^{(j)}(r)\right) .
\end{aligned}
$$

If the predicted intensity $v_{k \mid k-1}$ has the form

$$
v_{k \mid k-1}(\xi, r)=\sum_{i=1}^{J_{k \mid k-1}(r)} w_{k \mid k-1}^{(i)}(r) \mathcal{N}\left(\xi ; \Theta_{k \mid k-1}^{(i)}(r)\right) .
$$

Then the posterior intensity $v_{k}$ is given by

$$
v_{k}(\xi, r)=v_{k \mid k-1}(\xi, r)-v_{D, k}(\xi, r)+\sum_{z \in Z_{k}} v_{g, k}(\xi, r ; z),
$$

where

$$
\begin{aligned}
v_{D, k}(\xi, r) & =\sum_{i=1}^{J_{k \mid k-1}(r)} \sum_{l=0}^{J_{D, k}(r)} w_{D, k \mid k-1}^{(i, l)}(r) \mathcal{N}\left(\xi ; \Theta_{D, k \mid k-1}^{(i, l)}(r)\right), \\
w_{D, k \mid k-1}^{(i, l)}(r)= & w_{D, k}^{(l)}(r) w_{k \mid k-1}^{(i)}(r) q_{k \mid k-1}^{(i, l)}(r), \\
q_{D, k \mid k-1}^{(i, l)}(r)= & \mathcal{N}\left(m_{D, k}^{(l)}(r) ; \Pi\left(\Omega_{D, k}^{(l)}(r), \Theta_{k \mid k-1}^{(i)}(r)\right)\right), \quad q_{D, k \mid k-1}^{(i, 0)}(r)=1, \\
\Theta_{D, k \mid k-1}^{(i, l)}(r)= & \Psi\left(m_{D, k}^{(l)}(r), \Omega_{D, k}^{(l)}(r), \Theta_{k-1}^{(i)}(r)\right), \quad \Theta_{k \mid k-1}^{(i, 0)}(r)=\Theta_{k \mid k-1}^{(i)}(r), \\
v_{g, k}(\xi, r ; z)= & \sum_{i=1} \sum_{l=0} \sum_{j=1}^{J_{k \mid k-1}(r)} w_{g, k}^{(i, j, l)}(r ; z) \mathcal{N}\left(\xi ; \Theta_{g, k}^{(i, j, l)}(r ; z)\right), \\
w_{g, k}^{(i, j, l)}(r ; z)= & \frac{\kappa_{g, k}(z)+\sum_{D, k=1}^{(i, l)} \sum_{l=0}^{J_{k \mid k-1}(r) J_{D, k}(r) J_{g, k}(r)}(r) w_{g, k}^{(j)}(r) q_{g, k}^{(i, j, l)}(r ; z)}{J_{j=1}^{(i, l)}} w_{D, k \mid k-1}(r) w_{g, k}^{(j)}(r) q_{g, k}^{(i, j, l)}(r ; z) \\
q_{g, k}^{(i, j, l)}(r ; z)= & \mathcal{N}\left(z ; \Pi\left(\Omega_{g, k}^{(j)}(r), \Theta_{D, k \mid k-1}^{(i, l)}(r)\right)\right), \\
\Theta_{g, k}^{(i, j, l)}(r ; z)= & \Psi\left(z, \Omega_{g, k}^{(j)}(r), \Theta_{D, k \mid k-1}^{(i, l)}(r)\right) .
\end{aligned}
$$

Proof: For $v_{D, k}$, substituting (63), (65) into $p_{D, k}(x) v_{k \mid k-1}(x)$, and applying Lemma 2 to individual terms yields (67). For $v_{g, k}$, first substitute (67), (64) into the numerator of (47) and apply Lemma 2 to yield a (triple) sum of weighted Gaussians. Second, applying Lemma 1 to the integral in the denominator of (47) gives the (triple) sum in the denominator of (72). Combining the results for the numerator and denominator of (47) gives (71). 


\section{Simulation Results}

In this subsection we present simulation results for two examples to demonstrate the performance of the proposed PHD filter for LGJM models. For illustration purposes we consider a two-dimensional scenario where aircraft appear in the surveillance region $[-60,60] \times[-60,60] \mathrm{km}^{2}$. A single sensor located at $(0,0) \mathrm{km}$ provides position-only measurements to a controller. The interval between the samples is $T=5 s$ and the true number of aircraft at each sampling instant is not known.

During a level flight the aircraft dynamics can be modelled by a non-maneuver model and a maneuver model. Motion along a fixed heading at constant speed can be described by a non-maneuver model, for example, a constant velocity model. A level turn can be described by a maneuver model, for example, a co-ordinated turn model [2], [32]. The kinematic state of an aircraft is defined as

$$
\xi=\left[\begin{array}{llll}
p_{x}, & \dot{p}_{x}, & p_{y}, & \dot{p}_{y}
\end{array}\right]^{T}
$$

where $\left(p_{x}, p_{y}\right)$ denotes its Cartesian co-ordinates in the horizontal plane and $\left(\dot{p}_{x}, \dot{p}_{y}\right)$ denotes its velocities. The speed of the aircraft is in the range Mach [0.9, 1.1].

At a turn rate of $0^{\circ} s^{-1}$ the co-ordinated turn model reduces to the constant velocity model and the uniform motion of the aircraft can be modelled by the maneuver model. The aircraft motion models are described as follows. Model $r=1$ is a co-ordinated turn model with a turn rate of $0^{\circ} s^{-1}$ with linear Gaussian dynamics (17) given by

$$
\Omega_{f, k \mid k-1}(r=1)=\left(F_{k-1}(r=1), Q_{k}(r=1), 0\right),
$$

with

$$
F_{k-1}(r=1)=\left[\begin{array}{cccc}
1 & \frac{\sin \omega T}{\omega} & 0 & -\frac{1-\cos \omega T}{\omega} \\
0 & \cos \omega T & 0 & -\sin \omega T \\
0 & \frac{1-\cos \omega T}{\omega} & 1 & \frac{\sin \omega T}{\omega} \\
0 & \sin \omega T & 0 & \cos \omega T
\end{array}\right], \quad Q_{k}(r=1)=\sigma_{v_{1}}^{2}\left[\begin{array}{cccc}
\frac{T^{4}}{4} & \frac{T^{3}}{2} & 0 & 0 \\
\frac{T^{3}}{2} & T^{2} & 0 & 0 \\
0 & 0 & \frac{T^{4}}{4} & \frac{T^{3}}{2} \\
0 & 0 & \frac{T^{3}}{2} & T^{2}
\end{array}\right],
$$

where $\omega$ denotes turn rate. Perturbations in the lift and drag characteristics due to changes in the properties of the atmosphere are modelled as zero-mean Gaussian white noise with a standard deviation, $\sigma_{v_{1}}=$ $5 m s^{-2}$.

Model $r=2$ is a co-ordinated turn model with a counterclockwise turn rate of $3^{\circ} s^{-1}$ with standard deviation of noise, $\sigma_{v_{2}}=20 \mathrm{~m} \mathrm{~s}^{-2}$ to reflect the different noise characteristics during a level turn. Model $r=3$ is also a co-ordinated turn model but with a clockwise turn rate of $3^{\circ} s^{-1}$. The switching between 
the motion models is given by Markovian transition probability matrix as

$$
\left[t_{k \mid k-1}\left(r \mid r^{\prime}\right)\right]=\left[\begin{array}{ccc}
0.8 & 0.1 & 0.1 \\
0.1 & 0.8 & 0.1 \\
0.1 & 0.1 & 0.8
\end{array}\right] .
$$

The probability of target survival may change from one application to another and between different scenarios of an application. The reason is that in addition to some factors internal to the target, for example, aircraft altitude, fault-tolerance of instrumentation, fuel consumption and length of flight, target survival depends on certain external factors, for example, weather conditions. In general, the probability of target survival in military applications is lower than that in civilian applications where it may additionally depend on the maneuver an aircraft executes and the position of the aircraft relative to the location and type of threat (e.g. radar, anti-aircraft artillery, etc.) in the enemy surveillance region. A realistic model of the probability accounts for all of the above factors. In this paper, we do not cover modelling issues and assume that a model of the probability is given. Furthermore, the probability of target survival may be treated as a random variable and incorporated in the state vector to be estimated. However, for simplicity we assume that the probability is known. $p_{S, k \mid k-1}=0.99$ is assumed for modes $r^{\prime}=1,2,3$. Similarly, the probability of target detection may also vary depending on, for example, sensor characteristics, signal interference, weather conditions in civilian applications and in addition, countermeasures in military applications. A realistic model of the probability should consider these issues. Modelling issues are beyond the scope of the paper. We assume that such a model is given. In the examples that follow $p_{D, k}=0.98$ is assumed for modes $r=1,2,3$.

Measurements follow the observation model (18) given by

$$
\Omega_{g, k}=\left(H_{k}, R_{k}, 0\right)
$$

with

$$
H_{k}=\left[\begin{array}{cccc}
1 & 0 & 0 & 0 \\
0 & 0 & 1 & 0
\end{array}\right], \quad R_{k}=\sigma_{\epsilon}^{2} I_{2},
$$

where $I_{n}$ denotes a $n \times n$ identity matrix. The error in the sensor measurements is modelled as zero-mean Gaussian white noise with a standard deviation, $\sigma_{\epsilon}=40 \mathrm{~m}$. Clutter is modeled as a Poisson RFS with intensity

$$
\kappa_{k}(z)=\lambda_{c} V \mathcal{U}(z)
$$

where $\mathcal{U}(\cdot)$ denotes a uniform density over the surveillance region, $V=1.44 \times 10^{4} \mathrm{~km}^{2}$ is the volume of the surveillance region and $\lambda_{c}=3.47 \times 10^{-3} \mathrm{~km}^{-2}$ denotes the average number of clutter returns per 
unit volume.

The models for target births and spawnings are described next. Consider a scenario where the surveillance region includes three airport locations at $(40,-50) \mathrm{km},(-50,40) \mathrm{km}$ and $(-10,0) \mathrm{km}$. The intensity of the Poisson RFS of spontaneous births is given by

$$
\gamma_{k}(\xi, r)=0.1 \pi_{k}(r)\left[\mathcal{N}\left(\xi ; m_{\gamma}^{(1)}, P_{\gamma}\right)+\mathcal{N}\left(\xi ; m_{\gamma}^{(2)}, P_{\gamma}\right)+\mathcal{N}\left(\xi ; m_{\gamma}^{(3)}, P_{\gamma}\right)\right]
$$

with

$$
\begin{aligned}
& m_{\gamma}^{(1)}=\left[\begin{array}{llll}
4 \times 10^{4}, & 0, & -5 \times 10^{4}, & 0
\end{array}\right]^{T}, \\
& m_{\gamma}^{(2)}=\left[\begin{array}{lll}
-5 \times 10^{4}, & 0,4 \times 10^{4}, & 0
\end{array}\right]^{T} \text {, } \\
& m_{\gamma}^{(3)}=\left[\begin{array}{llll}
-1 \times 10^{4}, & 0, & 0, & 0
\end{array}\right]^{T} \text {, } \\
& P_{\gamma}=\operatorname{diag}\left(\left[10^{6}, 10^{4}, 10^{6}, 10^{4}\right]\right) \text {, }
\end{aligned}
$$

and the distribution of the models at birth is taken as

$$
\left[\pi_{k}(r)\right]=\left[\begin{array}{lll}
0.8, & 0.1, & 0.1
\end{array}\right] .
$$

Also consider the case where payloads originating from an aircraft contribute to sensor measurements, the intensity of the Poisson RFS of spawn births is given by

$$
\begin{aligned}
\beta_{k \mid k-1}\left(\xi, r \mid \xi^{\prime}, r^{\prime}\right) & =0.05 \pi_{k \mid k-1}\left(r \mid r^{\prime}\right) \mathcal{N}\left(\xi ; \xi^{\prime}, Q_{\beta}\right), \\
Q_{\beta} & =\operatorname{diag}\left(\left[\begin{array}{llll}
10^{4}, & 4 \times 10^{2}, & 10^{4}, & 4 \times 10^{2}
\end{array}\right]\right),
\end{aligned}
$$

and the distribution of the models for a given aircraft state is taken as

$$
\left[\pi_{k \mid k-1}\left(r \mid r^{\prime}\right)\right]=\left[\begin{array}{ccc}
0.8 & 0.1 & 0.1 \\
0.8 & 0.1 & 0.1 \\
0.8 & 0.1 & 0.1
\end{array}\right]
$$

For simplicity we assume the payload dynamics follow models $r=1,2,3$.

1) Example 1: At time $k=1$ an aircraft takes-off from $(-41,-51) \mathrm{km}$ and accelerates northwards. At time $k=3$ a second aircraft takes-off from $(-51,39) \mathrm{km}$ and accelerates towards $N 80^{\circ} \mathrm{E}$. A third aircraft takes-off from $(-9,1) \mathrm{km}$ at time $k=11$ and accelerates westwards. As the first aircraft initiates a counterclockwise turn at $k=31$ a payload separates from the aircraft and continues northwards. At time $k=44$ a payload separates from the second aircraft as it initiates a clockwise turn and continues along $S 70^{\circ} \mathrm{E}$. 
Fig. 1 shows the true aircraft and payload trajectories in the horizontal plane. A 1-D view of these trajectories along with the sensor measurements is shown in Fig. 2. Simulations show that the PHD filter works well even when the simulated data is not generated from the same models used by the filter. The position estimates of the PHD filter in Fig. 3 demonstrate that the filter provides accurate tracking performance in clutter. Since at each sampling instant the number of targets is not known the filter occasionally exhibits false estimates. However, as shown these estimates do not propagate with time.

The mean absolute error in the number of targets and the probability of track loss (see [16] for a definition of these measures), estimated from $10^{3}$ Monte Carlo runs, are shown in Fig. 4 for a position error radius of $50 \mathrm{~m}$.

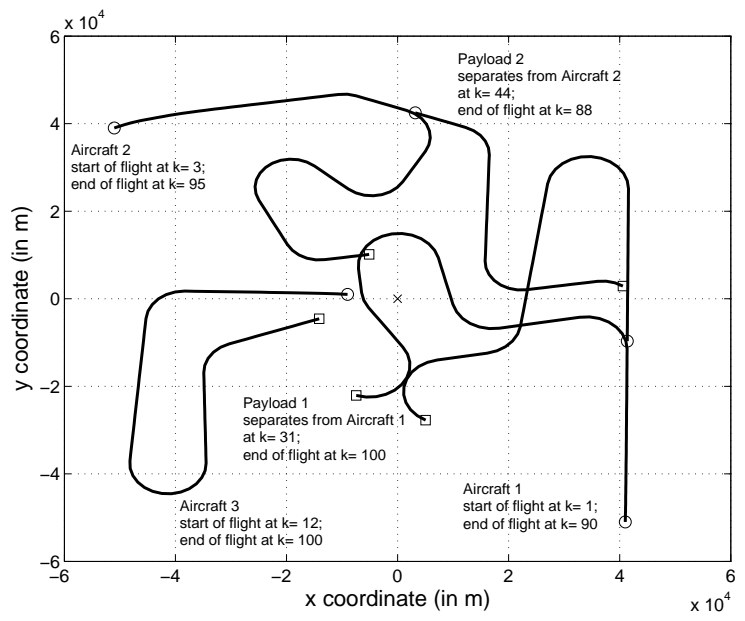

Fig. 1. Aircraft and payload trajectories. ' $\circ$ '- locations of start of flight; ' $\square$ '- locations of end of flight (' $\times$ '- location of sensor).

2) Example 2: At time $k=1$ three aircraft take-off simultaneously from the three airport locations. Aircraft 1 flies at a bearing of $N 45^{\circ} \mathrm{W}$ from $(-41,-51) \mathrm{km}$, aircraft 2 flies eastwards from $(-51,39) \mathrm{km}$ and aircraft 3 flies at a bearing of $S 45^{\circ} \mathrm{E}$ from $(-9,1) \mathrm{km}$. Assuming all three aircraft exist at each sampling instant and no other targets appear in the surveillance region, the performance of the proposed PHD filter can be compared with that of the well-known IMMJPDA filter which tracks a fixed and known number of targets.

As indicated previously the PHD filter has a complexity of $\mathcal{O}\left(J_{k-1}\left|Z_{k}\right|\right)$ where $J_{k-1}$ is the number of Gaussian components representing $v_{k-1}$ for a fixed model $r^{\prime}$ at time $k-1$ and $\left|Z_{k}\right|$ denotes the number of measurements at time $k$. Computationally efficient implementation of data association in JPDA has been the subject of much research. Exploiting parallel implementation, the column-recursive algorithm 

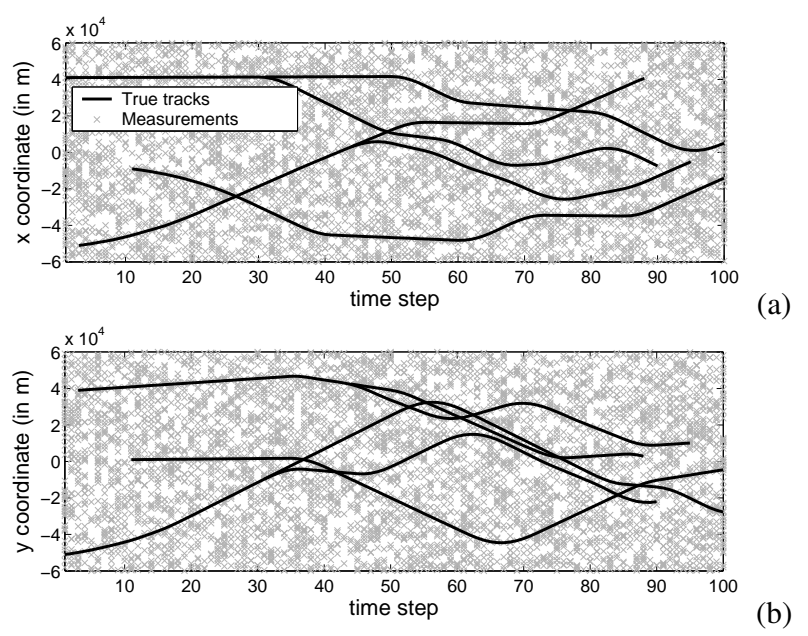

Fig. 2. Measurement data and true target positions.
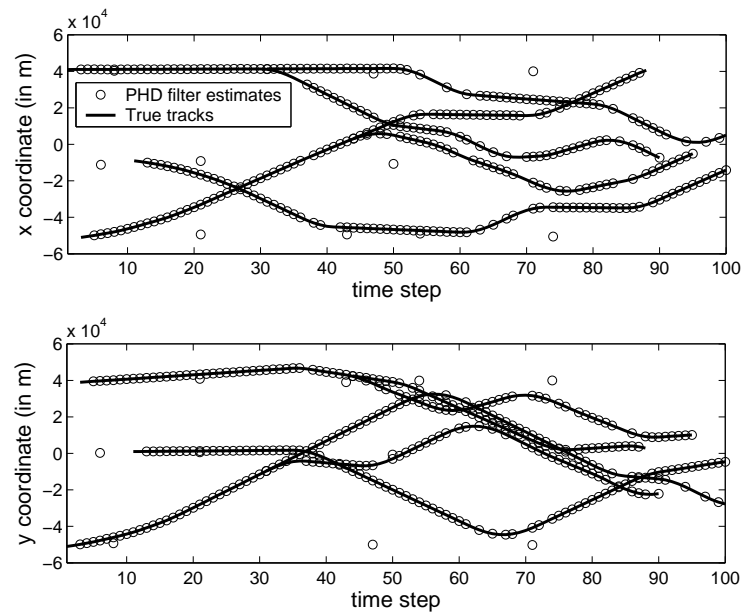

(a)

Fig. 3. Position estimates of the Gaussian mixture PHD filter.

CR-JPDA [33] has a complexity of $\mathcal{O}\left(N\left|Z_{k}\right|^{2} 2^{N}\right)$ for $\mathrm{N}$ targets.

Fig. 5 shows the trajectories of the three aircraft. Fig. 6 (a) shows the mean absolute error in the estimate of the number of aircraft by the PHD filter. Fig. 6 (b) shows the probability of track loss at various clutter rates while a comparison of the averaged CPU time involved at each step for the two filters is shown in Fig. 6 (c). Simulation results obtained from $10^{3}$ Monte Carlo runs indicate that at any given clutter rate the tracking performance of the PHD filter is similar to that of the IMMJPDA filter at lower computational complexity.

Fig. 7 shows the tracking performance of the Gaussian mixture PHD filter versus the probability of 

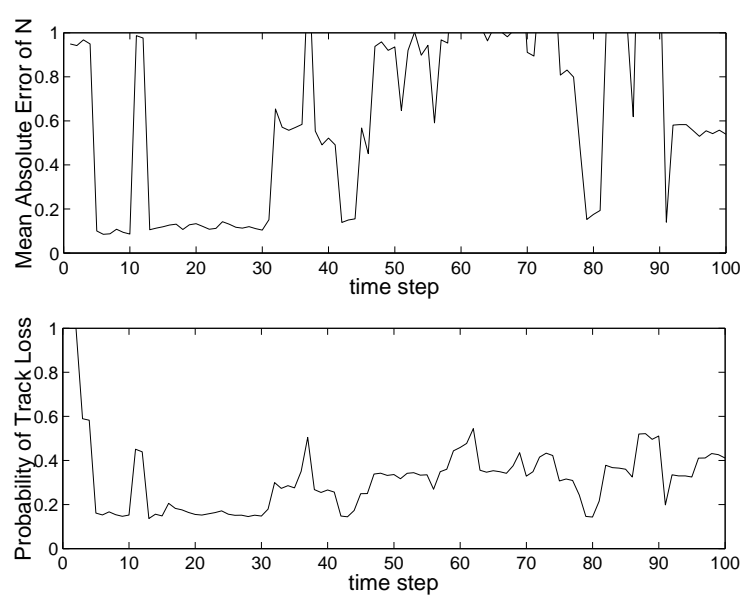

(a)

Fig. 4. Mean absolute error of estimated number of targets and probability of track lost.

target detection $p_{D, k}$ in the range $[0.7,1.0]$ with a fixed clutter rate $\lambda_{c}=3.47 \times 10^{-3} \mathrm{~km}^{-2}$. Fig. 7 (a) shows the mean absolute error in the estimate of the number of aircraft by the PHD filter. However, a comparison with the performance of the IMMJPDA filter is more intuitive. This result is remarkable because the PHD filter must resolve detection uncertainty in addition to the uncertainty in the number of targets and therefore is expected to perform poorly with increasing uncertainty in the number of targets due to increasing detection uncertainty. However, as shown in Figs. 7 (b) and (c) the tracking performance of the Gaussian mixture PHD filter is very similar to that of the IMMJPDA filter at a much lower computational cost.

\section{THE PHD FILTER FOR NONLINEAR GJM MULTI-TARGET MODELS}

A JMS comprising of nonlinear models accommodates an even wider range of applications by providing a greater generality for modelling systems that switch between various models. Extension of the PHD filter for nonlinear models relaxes assumption A.4 and the state transition density and likelihood functions take the form

$$
\begin{aligned}
f_{k \mid k-1}\left(\xi, r \mid \xi^{\prime}, r^{\prime}\right) & =\mathcal{N}\left(\xi, F_{k-1}\left(\xi^{\prime}, r\right), Q_{k}(r)\right) t_{k \mid k-1}\left(r \mid r^{\prime}\right) \\
g_{k}(z \mid \xi, r) & =\mathcal{N}\left(z, H_{k}(\xi, r), R_{k}(r)\right)
\end{aligned}
$$

where $F_{k-1}(\cdot, r)$ and $H_{k}(\cdot, r)$ denote nonlinear functions of model $r$. The contribution of the intensity term due to the motion of the targets $v_{f, k \mid k-1}(\xi, r)$ to the predicted intensity at time $k$ in (30) for a given 


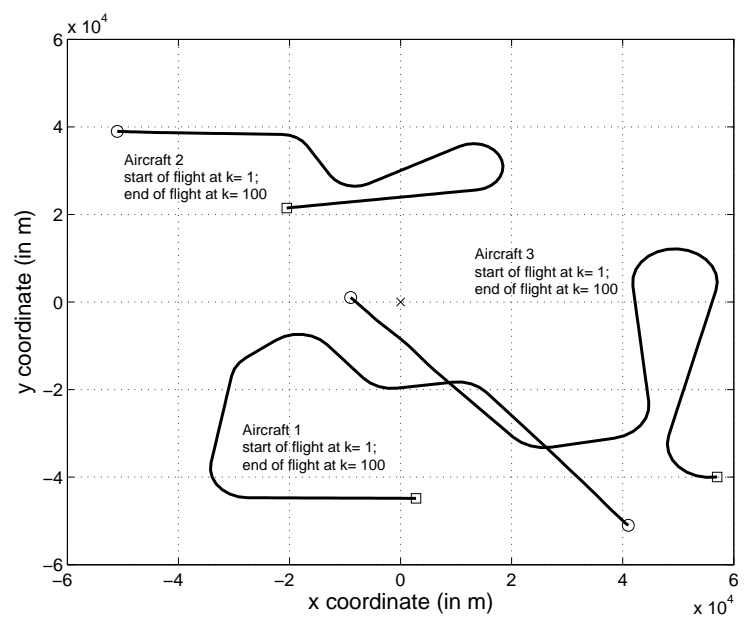

Fig. 5. Aircraft and payload trajectories. ' $\circ$ '- locations of start of flight; ' $\square$ '- locations of end of flight (' $\times$ ' - location of sensor).

prior intensity $v_{k-1}$ is given by

$$
v_{f, k \mid k-1}(\xi, r)=\sum_{r^{\prime}} p_{S, k \mid k-1}(r) t_{k \mid k-1}\left(r \mid r^{\prime}\right) \int \mathcal{N}\left(\xi ; F_{k-1}\left(\xi^{\prime}, r\right), Q_{k}(r)\right) v_{k-1}\left(\xi^{\prime}, r^{\prime}\right) d \xi^{\prime}
$$

Since $F_{k-1}(\cdot, r)$ is a nonlinear function, $v_{f, k \mid k-1}(\xi, r)$ does not admit a closed form. The predicted intensity $v_{k \mid k-1}(\xi, r)$ at time $k$ is a weighted sum of various functions of $\xi$, many of which are nonGaussian due to $v_{f, k \mid k-1}(\xi, r)$.

Similarly, the contribution of the intensity term due to the detected targets $v_{g, k}(\xi, r)$ to the posterior intensity at time $k$ in (42) for a given predicted intensity of Gaussian mixture form is given by

$$
v_{g, k}(\xi, r)=\frac{p_{D, k}(r) \mathcal{N}\left(z ; H_{k}(\xi, r)\right) v_{k \mid k-1}(\xi, r)}{\kappa_{k}(z)+\sum_{r^{\prime}} p_{D, k}\left(r^{\prime}\right) \int \mathcal{N}\left(z ; H_{k}\left(\xi^{\prime}, r^{\prime}\right)\right) v_{k \mid k-1}\left(\xi^{\prime}, r^{\prime}\right) d \xi^{\prime}} .
$$

Since $H_{k}(\cdot, r)$ is a nonlinear function, $v_{g, k}(\xi, r)$ does not admit a closed form and the posterior intensity $v_{k}(\xi, r)$ at time $k$ comprises of non-Gaussian components due to $v_{g, k}(\xi, r)$. At present there exists no tractable analytic method for tracking multiple targets with nonlinear jump Markov dynamics. In this section we present an analytic approximation of the PHD recursion.

In single target filtering, analytic approximations of the nonlinear Bayes filter include the extended Kalman filter (EKF) and the unscented Kalman filter (UKF) [34]. The EKF approximates the posterior density by a Gaussian, which is propagated in time by applying Kalman recursions to local linearizations of the (nonlinear) mappings $F_{k-1}(\cdot, r)$ and $H_{k}(\cdot, r)$. The UKF also approximates the posterior density 


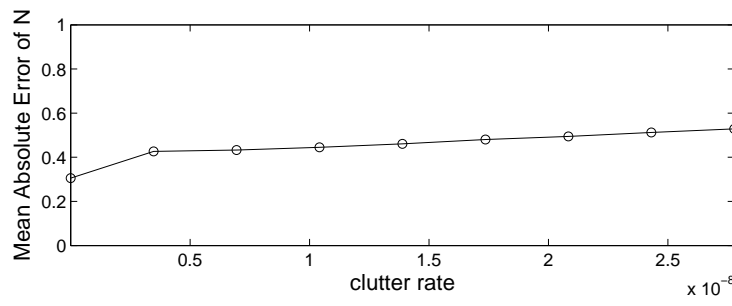

(a)

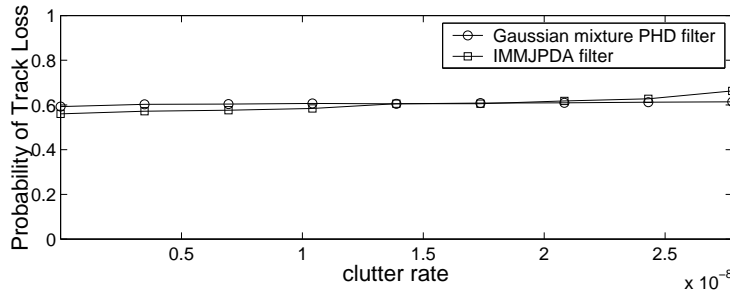

10 (b)

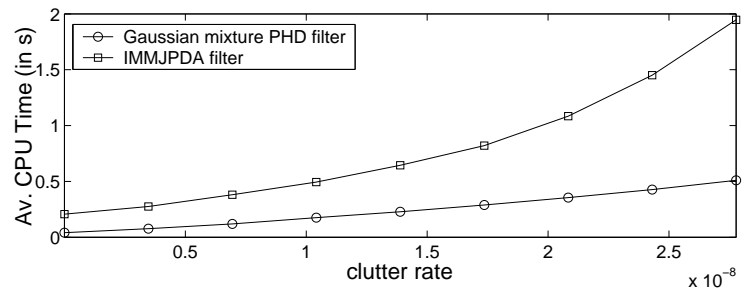

(c)

Fig. 6. Tracking performance and computational complexity versus clutter rate for $p_{D, k}=0.98$ and CPEP radius $=50 \mathrm{~m}$.

by a Gaussian, but instead of using the linearized model, it computes the Gaussian approximation of the posterior density at the next time step using the unscented transform.

The EKF and UKF approximations of the posterior density can be derived exactly using the linear regression Kalman filter (LRKF) [35] which approximates the nonlinear process and measurement functions using statistical linear regression through some regression points. The EKF is derived using a single regression point only while the UKF is derived using $p=2(n+\kappa)$ regression points for an $n$-dimensional kinematic state $\xi$ where $\kappa$ is a degree of freedom in the choice of the regression points [34]. It has been shown that in most applications approximations using the unscented transform are more accurate [18]. In the following part of this section we discuss linear approximations of $F_{k-1}(\cdot, r)$ and $H_{k}(\cdot, r)$ using the UKF and demonstrate the performance of the PHD filter for nonlinear models through a simulation example.

Consider the nonlinear function $F_{k-1}(\cdot, r)$ evaluated in $p$ points $\left(\mathcal{X}_{k-1, i}(r), \mathcal{Y}_{k-1, i}(r)\right), i=1, \ldots, p$ 

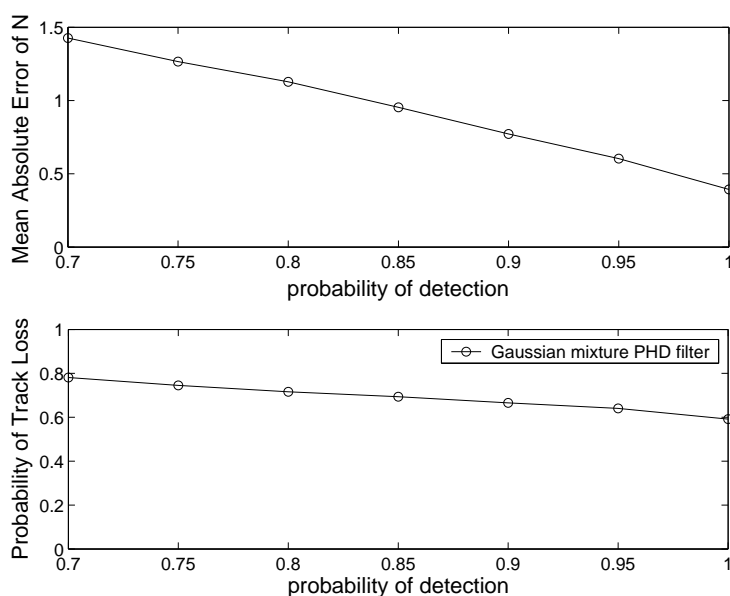

(a)

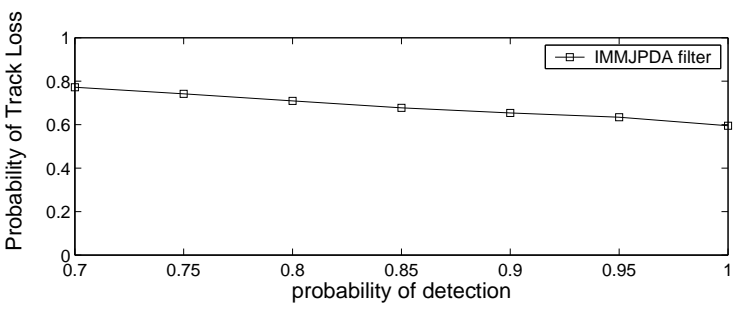

(b)

Fig. 7. Tracking performance versus probability of detection for $\lambda_{c}=3.47 \times 10^{-3} \mathrm{~km}^{-2}$ and CPEP radius $=50 \mathrm{~m}$.

around $\hat{m}_{k-1}(r)$ where $\mathcal{Y}_{k-1, i}(r)=F_{k-1}\left(\mathcal{X}_{k-1, i}(r), r\right), i=1, \ldots, p$ such that

$$
\begin{aligned}
\hat{m}_{k-1}(r) & =\frac{1}{p} \sum_{i=1}^{p} \mathcal{X}_{k-1, i}(r) \\
\hat{P}_{k-1}(r) & =\frac{1}{p} \sum_{i=1}^{p}\left(\mathcal{X}_{k-1, i}(r)-\hat{m}_{k-1}(r)\right)\left(\mathcal{X}_{k-1, i}(r)-\hat{m}_{k-1}(r)\right)^{T} .
\end{aligned}
$$

A choice of regression points satisfying (95) is $2 \kappa$ points $\mathcal{X}_{k-1,0}(r), n$ points $\mathcal{X}_{k-1, i}(r)$ and $n$ points $\mathcal{X}_{k-1, n+i}(r)$ with

$$
\begin{aligned}
\mathcal{X}_{k-1,0}(r) & =\hat{m}_{k-1}(r), \\
\mathcal{X}_{k-1, i}(r) & =\hat{m}_{k-1}(r)+\sqrt{\frac{p}{2}} \hat{P}_{k-1, i}(r), \\
\mathcal{X}_{k-1, n+i}(r) & =\hat{m}_{k-1}(r)-\sqrt{\frac{p}{2}} \hat{P}_{k-1, i}(r),
\end{aligned}
$$

where $\hat{P}_{k-1, i}(r)$ denotes the column vector such that $\hat{P}_{k-1}(r)=\sum_{i} \hat{P}_{k-1, i}(r) \hat{P}_{k-1, i}^{T}(r)$.

The statistical linear regression of $F_{k-1}(\cdot, r)$ around $\hat{m}_{k-1}(r)$ is the function $A_{k-1}(r) m+b_{k-1}(r)$ 
derived from the minimization of the sum of the squared errors

$$
\left(A_{k-1}(r), b_{k-1}(r)\right)=\arg \min _{A(r), b(r)} \frac{1}{p} \sum_{i=1}^{p}\left\|e_{k \mid k-1, i}(r)\right\|^{2},
$$

with $e_{k \mid k-1, i}(r)=\mathcal{Y}_{k-1, i}(r)-\left(A_{k-1}(r) \mathcal{X}_{k-1, i}(r)+b_{k-1}(r)\right)$. The solution of (97) can be shown to be

$$
\begin{aligned}
& A_{k-1}(r)=P_{k-1, x y}^{T}(r) \hat{P}_{k-1}^{-1}(r), \\
& b_{k-1}(r)=\bar{y}_{k-1}(r)-A_{k-1}(r) \hat{m}_{k-1}(r),
\end{aligned}
$$

and the covariance of the error $e_{k \mid k-1, i}$ as

$$
P_{k-1, p e}(r)=\frac{1}{p} \sum_{i=1}^{p}\left\|e_{k \mid k-1, i}(r)\right\|^{2}=P_{k-1, y y}(r)-A_{k-1}(r) \hat{P}_{k-1}(r) A_{k-1}^{T}(r),
$$

where

$$
\begin{aligned}
\bar{y}_{k-1}(r) & =\frac{1}{p} \sum_{i=1}^{p} \mathcal{Y}_{k-1, i}(r), \\
P_{k-1, y y}(r) & =\frac{1}{p} \sum_{i=1}^{p}\left(\mathcal{Y}_{k-1, i}(r)-\bar{y}_{k-1}(r)\right)\left(\mathcal{Y}_{k-1, i}(r)-\bar{y}_{k-1}(r)\right)^{T}, \\
P_{k-1, x y}(r) & =\frac{1}{p} \sum_{i=1}^{p}\left(\mathcal{X}_{k-1, i}(r)-\hat{m}_{k-1}(r)\right)\left(\mathcal{Y}_{k-1, i}(r)-\bar{y}_{k-1}(r)\right)^{T} .
\end{aligned}
$$

Admitting the following approximation in (91)

$$
\mathcal{N}\left(\xi ; F_{k-1}\left(\xi^{\prime}, r\right), Q_{k}(r)\right) \approx \mathcal{N}\left(\xi, A_{k-1}(r) \xi^{\prime}+b_{k-1}(r), P_{k-1, p e}(r)+Q_{k}(r)\right),
$$

Lemma 1 can be applied in (93) to obtain $v_{f, k \mid k-1}$ expressed in Gaussian mixture form.

Similarly, $H_{k}(\cdot, r)$ can be evaluated in $p$ points $\left(\mathcal{X}_{k \mid k-1, j}(r), \mathcal{Z}_{k, j}(r)\right), j=1, \ldots, p$ around $\hat{m}_{k \mid k-1}(r)$ where $\mathcal{Z}_{k, j}(r)=H_{k}\left(\mathcal{X}_{k \mid k-1, j}(r), r\right), j=1, \ldots, p$. The statistical linear regression of $H_{k}(\cdot, r)$ around $\hat{m}_{k \mid k-1}(r)$ is the function $C_{k}(r) m+d_{k}(r)$ derived as

$$
\left(C_{k}(r), d_{k}(r)\right)=\arg \min _{C(r), d(r)} \frac{1}{p} \sum_{j=1}^{p}\left\|e_{k, j}(r)\right\|^{2},
$$

with $e_{k, j}(r)=\mathcal{Z}_{k, j}(r)-\left(C_{k}(r) \mathcal{X}_{k \mid k-1, j}(r)+d_{k}(r)\right)$. The solution of (103) can be shown to be

$$
\begin{aligned}
C_{k}(r) & =P_{k, x z}^{T}(r) \hat{M}_{k \mid k-1}^{-1}(r), \\
d_{k}(r) & =\bar{z}_{k}(r)-C_{k}(r) \hat{m}_{k \mid k-1}(r),
\end{aligned}
$$

and

$$
P_{k, m e}(r)=\frac{1}{p} \sum_{j=1}^{p}\left\|e_{k, j}(r)\right\|^{2}=P_{k-1, z z}(r)-C_{k}(r) \hat{M}_{k \mid k-1}(r) C_{k}^{T}(r),
$$


where

$$
\begin{aligned}
\bar{z}_{k}(r) & =\frac{1}{p} \sum_{j=1}^{p} \mathcal{Z}_{k, j}(r), \\
P_{k, z z}(r) & =\frac{1}{p} \sum_{j=1}^{p}\left(\mathcal{Z}_{k, j}(r)-\bar{z}_{k}(r)\right)\left(\mathcal{Z}_{k, j}(r)-\bar{z}_{k}(r)\right)^{T}, \\
P_{k, x z}(r) & =\frac{1}{p} \sum_{j=1}^{p}\left(\mathcal{X}_{k \mid k-1, j}(r)-\hat{m}_{k \mid k-1}(r)\right)\left(\mathcal{Z}_{k, j}(r)-\bar{z}_{k}(r)\right)^{T} .
\end{aligned}
$$

Admitting the following approximation in (92)

$$
\mathcal{N}\left(z ; H_{k}(\xi, r), R_{k}(r)\right) \approx \mathcal{N}\left(z, C_{k}(r) \xi+d_{k}(r), P_{k, m e}(r)+R_{k}(r)\right)
$$

Lemma 1 and 2 can be applied in (94) to obtain $v_{g, k}$ in Gaussian mixture form.

Note that for nonlinear jump Markov spontaneous birth and spawn models each non-Gaussian constituent function of the mixture models can be approximated by a Gaussian using the linear approximation method described above. The expressions for the PHD recursion are notationally cumbersome and therefore omitted.

\section{A. Simulation Results}

In this subsection we demonstrate the performance of the proposed PHD filter for nonlinear Gaussian jump Markov models. Assuming the turn rate is not a known constant the maneuver model becomes a nonlinear one. Augmenting the state vector to estimate the turn rate, the kinematic state of the aircraft is defined as

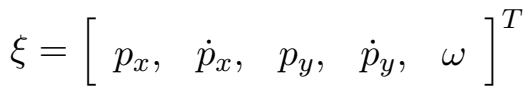

The motion models are as follows. Model $r=1$ is a co-ordinated turn model with a known turn rate of $0^{\circ} \mathrm{s}^{-1}$ and standard deviation of process noise, $\sigma_{v_{1}}=5 \mathrm{~m} \mathrm{~s}^{-2}$. Model $r=2$ is a co-ordinated turn model with an unknown turn rate given by

$$
F_{k-1}(\omega, r=2)=\left[\begin{array}{ccccc}
1 & \frac{\sin \omega T}{\omega} & 0 & -\frac{1-\cos \omega T}{\omega} & 0 \\
0 & \cos \omega T & 0 & -\sin \omega T & 0 \\
0 & \frac{1-\cos \omega T}{\omega} & 1 & \frac{\sin \omega T}{\omega} & 0 \\
0 & \sin \omega T & 0 & \cos \omega T & 0 \\
0 & 0 & 0 & 0 & 1
\end{array}\right], Q_{k}(r=2)=\sigma_{v_{2}}^{2}\left[\begin{array}{ccccc}
\frac{T^{4}}{4} & \frac{T^{3}}{2} & 0 & 0 & 0 \\
\frac{T^{3}}{2} & T^{2} & 0 & 0 & 0 \\
0 & 0 & \frac{T^{4}}{4} & \frac{T^{3}}{2} & 0 \\
0 & 0 & \frac{T^{3}}{2} & T^{2} & 0 \\
0 & 0 & 0 & 0 & T^{2}
\end{array}\right]
$$


and a process noise standard deviation of $10 \mathrm{~m} \mathrm{~s}^{-2}$ and $0.5^{\circ} \mathrm{s}^{-2}$ for the linear and turn portions respectively. The Markovian transition probability matrix is taken as

$$
\left[t_{k \mid k-1}\left(r \mid r^{\prime}\right)\right]=\left[\begin{array}{cc}
0.8 & 0.2 \\
0.2 & 0.8
\end{array}\right]
$$

Aircraft are observed by a sensor providing bearing and range measurements in the region $[-\pi, \pi] \operatorname{rad} \times$ $[0,60] \mathrm{km}$. The measurements are given by

$$
z=\left[\begin{array}{c}
\arctan \left(p_{y} / p_{x}\right) \\
\sqrt{p_{x}^{2}+p_{y}^{2}}
\end{array}\right]+\epsilon_{k}
$$

where $\epsilon_{k} \sim \mathcal{N}\left(\cdot ; 0, R_{k}\right)$ with $R_{k}=\operatorname{diag}\left(\left[\sigma_{\theta}^{2}, \sigma_{r}^{2}\right]\right), \sigma_{\theta}=(\pi / 180) \mathrm{rads}^{-1}$ and $\sigma_{r}=10 \mathrm{~m}$. The average number of clutter returns per unit volume is $\lambda_{c}=1.326 \times 10^{-1}(\mathrm{rad} \mathrm{km})^{-1}$.

The models for the births and spawnings are described as follows. The surveillance region includes three airport locations at $(40,-50) \mathrm{km},(-50,40) \mathrm{km}$ and $(-10,-10) \mathrm{km}$. The intensity of the Poisson RFS of spontaneous births is given by

$$
\gamma_{k}(\xi, r)=0.1 \pi_{k}(r)\left[\mathcal{N}\left(\xi ; m_{\gamma}^{(1)}, P_{\gamma}\right)+\mathcal{N}\left(\xi ; m_{\gamma}^{(2)}, P_{\gamma}\right)+\mathcal{N}\left(\xi ; m_{\gamma}^{(3)}, P_{\gamma}\right)\right]
$$

with

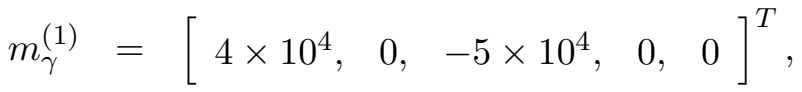

$$
\begin{aligned}
& m_{\gamma}^{(2)}=\left[\begin{array}{llll}
-5 \times 10^{4}, & 0,4 \times 10^{4}, & 0, & 0
\end{array}\right]^{T},
\end{aligned}
$$

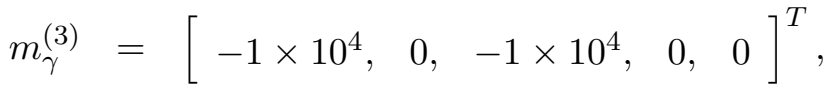

$$
\begin{aligned}
& P_{\gamma}=\operatorname{diag}\left(\left[10^{6}, 10^{4}, 10^{6}, 10^{4}, 10^{-8}\right]\right) \text {, }
\end{aligned}
$$

and the distribution of the models at birth is taken as

$$
\left[\pi_{k}(r)\right]=\left[\begin{array}{ll}
0.8, & 0.2
\end{array}\right]
$$

The intensity of the Poisson RFS of spawn births is given by

$$
\begin{aligned}
& \beta_{k \mid k-1}\left(\xi, r \mid \xi^{\prime}, r^{\prime}\right)=0.05 \pi_{k \mid k-1}\left(r \mid r^{\prime}\right) \mathcal{N}\left(\xi ; \xi^{\prime}, Q_{\beta}\right), \\
& Q_{\beta}=\operatorname{diag}\left(\left[10^{4}, 4 \times 10^{2}, 10^{4}, 4 \times 10^{2}, 10^{-8}\right]\right),
\end{aligned}
$$


and the distribution of the models for a given aircraft state is taken as

$$
\left[\pi_{k \mid k-1}\left(r \mid r^{\prime}\right)\right]=\left[\begin{array}{cc}
0.8 & 0.2 \\
0.8 & 0.2
\end{array}\right] .
$$

The settings for all other parameters are identical to those in section III-D.

At time $k=1$ an aircraft takes-off from $(-41,-51) \mathrm{km}$ and accelerates northwards. At time $k=31$ the aircraft executes a clockwise turn through $45^{\circ}$ at $1^{\circ} s^{-1} .30 s$ later the aircraft executes a $2^{\circ} s^{-1}$ counterclockwise turn. The aircraft then executes a $1^{\circ} s^{-1}$ clockwise turn at time $k=70$. At time $k=3$ a second aircraft takes-off from $(-51,39) \mathrm{km}$ and accelerates at a bearing of $N 80^{\circ} \mathrm{E}$. The aircraft executes two clockwise turns at $1^{\circ} s^{-1}$ and $2^{\circ} s^{-1}$ and flies at a heading of $S 60^{\circ} \mathrm{W}$ for $55 \mathrm{~s}$ before executing a $90^{\circ}$ counterclockwise turn at $2^{\circ} \mathrm{s}^{-1}$. A third aircraft takes-off from $(-9,-11) \mathrm{km}$ at time $k=12$ and accelerates along the initial heading of $S 80^{\circ} \mathrm{W}$. At time $k=35$ the aircraft performs a $180^{\circ}$ counterclockwise maneuver at $1^{\circ} s^{-1}$ followed by a sequence of clockwise and counterclockwise maneuvers at $2^{\circ} s^{-1}$. Two payloads separate from Aircraft 1 and Aircraft 2 at time $k=31$ and $k=56$ respectively and continue until $k=100$.

Fig. 8 shows the true trajectories in the horizontal plane. As shown in Fig. 9 the proposed PHD filter provides reasonably accurate position estimates at most times. Fig. 10 shows the mean absolute error in the number of targets and the probability of track loss for a position error radius of $50 \mathrm{~m}$ estimated from $10^{3}$ Monte Carlo runs.

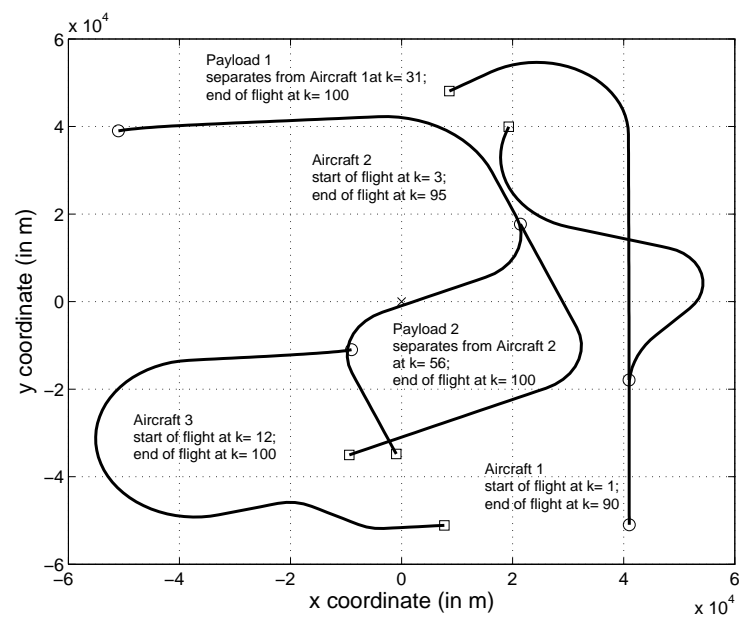

Fig. 8. Aircraft and payload trajectories. ' $\circ$ '- locations of start of flight; ' $\square$ '- locations of end of flight (' $\times$ '- location of sensor). 

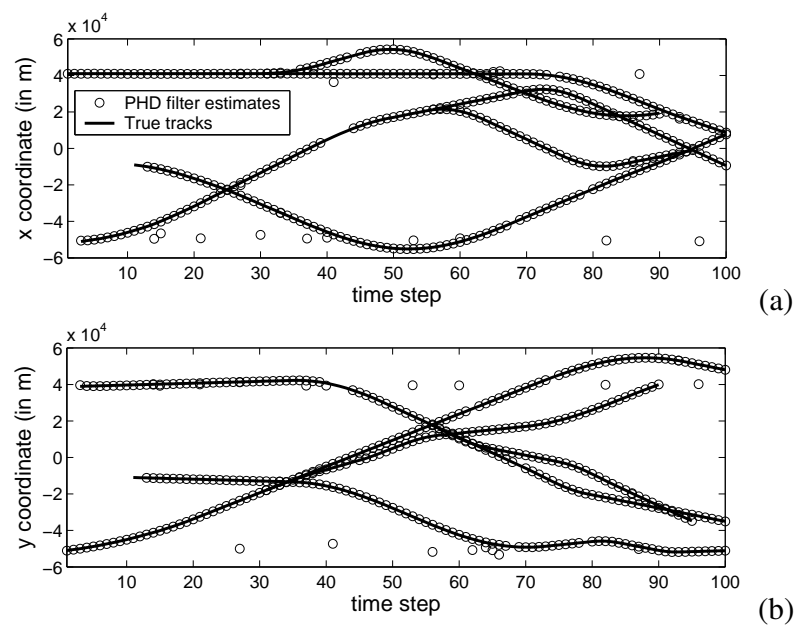

(a)

Fig. 9. Position estimates of the Gaussian mixture PHD filter.
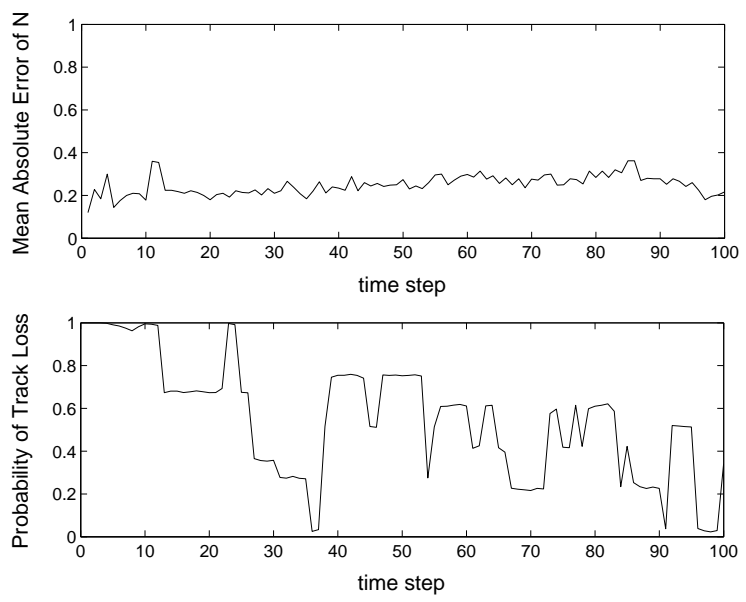

Fig. 10. Mean absolute error of estimated number of targets and probability of track lost.

\section{CONCLUSiOns}

A multi-target model that accommodates births, deaths and switching linear Gaussian dynamics has been proposed. For this so-called linear Gaussian jump Markov system (LGJMS) multi-target model, a closed form solution to the PHD recursion has been derived. Based on this solution, an efficient algorithm that can track an unknown, time-varying number of maneuvering targets in clutter has been developed. Extension of this algorithm to track maneuvering targets with non-linear jump Markov dynamics has also been proposed. The proposed approach is applicable to a general class of models expedient for a range of practical applications in multi-target tracking that are deemed intractable using conventional 
techniques. Simulations have demonstrated the effectiveness of the proposed multi-target filters for tracking an unknown and time-varying number of maneuvering targets in clutter and detection uncertainty. In comparison with the well-known IMMJPDA filter, the proposed approach exhibits an unprecedented combination of good tracking performance and high computational efficiency.

\section{REFERENCES}

[1] Y. Bar-Shalom and T. E. Fortmann, Tracking and Data Association. Academic Press, San Diego, 1988.

[2] Y. Bar-Shalom, X.-R. Li, and T. Kirubarajan, Estimation with Application to Tracking and Navigation. Wiley, 2001.

[3] X.-R. Li and V. Jilkov, "Survey of maneuvering target tracking. Part 1: Dynamic models," IEEE Trans. AES, vol. 39, no. 4, pp. 1333-1364, 2003.

[4] X.-R. Li and V. Jilkov, "Survey of maneuvering target tracking. Part V: Multiple-model methods," IEEE Trans. AES, vol. 41, no. 4, pp. 1255-1321, 2005.

[5] H. Blom and Y. Bar-Shalom, "The interacting multiple model algorithm for systems with Markovian switching coefficients," IEEE Trans. AC, vol. AC-33, pp. 780-783, 1988.

[6] Y. Bar-Shalom, K. C. Chang, and H. Blom, "Tracking splitting targets in clutter by using an Interactive Multiple Model Joint Probabilistic Data Association filter," Multitarget Multisensor Tracking: Applications and Advances, Artech House, vol. II, pp. 93-110, 1992.

[7] H. Blom and E. Bloem, "Combining IMM and JPDA for tracking multiple maneuvering targets in clutter," in Proc. Int'l Conf. on Information Fusion, Cairns, Australia, vol. 1, pp. 705-712, 2002.

[8] J. Tugnait, "Tracking of multiple maneuvering targets in clutter using multiple sensors, IMM, and JPDA coupled filtering," IEEE Trans. AES, vol. 40, no. 1, pp. 320-330, 2004.

[9] S. Jeong and J. K. Tugnait, "Tracking of multiple maneuvering targets in clutter with possibly unresolved measurements using IMM and JPDAM coupled filtering," in Proc. of the American Control Conference, pp. 1257-1262, 2005.

[10] S. B. R.J. Dempster and T. Nichols, "Combining IMM filtering and MHT data association for multitarget tracking," in Proc. of the Twenty-Ninth Southeastern Symposium, 1997.

[11] W. Koch, "Fixed-interval retrodiction approach to Bayesian IMM-MHT for maneuvering multiple targets," IEEE Trans. AES, vol. 36, no. 1, pp. 2-14, 2000.

[12] R. Mahler, "Multi-target Bayes filtering via first-order multi-target moments," IEEE Trans. AES, vol. 39, no. 4, pp. 1152$1178,2003$.

[13] I. Goodman, R. Mahler, and H. Nguyen, Mathematics of Data Fusion. Kluwer Academic Publishers, 1997.

[14] B. Vo, S. Singh, and A. Doucet, "Sequential Monte Carlo implementation of the PHD filter for multi-target tracking," in Proc. Int'l Conf. on Information Fusion, Cairns, Australia, pp. 792-799, 2003.

[15] B. Vo, S. Singh and A. Doucet, "Sequential Monte Carlo methods for multi-target filtering with random finite sets," in IEEE Trans. AES, vol. 41, no. 4, pp. 1224-1245, 2005, also: http://www.ee.unimelb.edu.au/staff/bv/index.html.

[16] B. Vo and W. K. Ma, “The Gaussian mixture Probability Hypothesis Density filter," IEEE Trans. Signal Processing, vol. 54, no. 11, pp. 4091-4104, 2006, also: http://www.ee.unimelb.edu.au/staff/bv/index.html.

[17] B. Vo and W. K. Ma, “A closed-form solution to the Probability Hypothesis Density filter," in Proc. Int'l Conf. on Information Fusion, Philadelphia, 2005. 
[18] S. J. Julier and J. K. Uhlmann, “Unscented filtering and nonlinear estimation,” in Proc. IEEE, vol. 92, no. 3, pp. 401-422, 2004.

[19] R. Mahler, "An introduction to multisource-multitarget statistics and applications," Lockheed Martin Technical Monograph, 2000.

[20] D. Daley and D. Vere-Jones, An Introduction to the Theory of Point Processes. Springer-Verlag, 1988.

[21] H. Sidenbladh and S. Wirkander, "Tracking random sets of vehicles in terrain," in Proc. 2003 IEEE Workshop on MultiObject Tracking, Madison Wisconsin, 2003.

[22] B. Vo, W. K. Ma, and S. Singh, "Locating an unknown time-varying number of speakers: A Bayesian random finite set approach,” in Proc. 2005 IEEE Int. Conf. Acoust., Speech, Signal Processing, Philadelphia, vol. 4, pp. 1073-1076, 2005.

[23] W. K. Ma, B. Vo, S. Singh, and A. Baddeley, "Tracking an unknown and time varying number of speakers using TDOA measurements: A random finite set approach,” IEEE Trans. Signal Processing, vol. 54, no. 9, pp. 3291-3304, 2006.

[24] S. Blackman, Multiple Target Tracking with Radar Applications. Artech House, Norwood, 1986.

[25] A. Johansen, S. Singh, A. Doucet, and B. Vo, "Convergence of the sequential Monte Carlo implementation of the PHD filter," to appear in Methodology and Computing in Applied Probability, 2006.

[26] D. Clark and J. Bell, "Bayesian multiple target tracking in forward scan sonar images using the PHD filter," in IEE Radar, Sonar and Navigation, vol. 152, no. 5, pp. 327-334, October 2005.

[27] B. Vo and W. K. Ma, "Joint detection and tracking of multiple maneuvering targets using random finite sets," in Proc. ICARCV, Kunming, China, 2004.

[28] K. Punithakumar, T. Kirubarajan, and A. Sinha, "A multiple model Probability Hypothesis Density filter for tracking maneuvering targets," in O. E. Drummond (ed.) Signal and Data Processing of Small Targets, Proc. SPIE, vol. 5428, pp. 113-121, 2004.

[29] D. Stoyan, D. Kendall, and J. Mecke, Stochastic Geometry and its Applications. John Wiley \& Sons, 1995.

[30] J. F. C. Kingman, Poisson Processes. Oxford University Press, Oxford, 1993.

[31] D. Reid, “An algorithm for tracking multiple targets,” IEEE Trans. AC, vol. AC-24, no. 6, pp. 843-854, 1979.

[32] Y. Bar-Shalom and X.-R. Li, Multitarget-Multisensor Tracking: Principles and Techniques. Storrs, CT: YBS Publishing, 1995.

[33] L. Nyland, J. Prins, A. Goldberg, P. Mills, J. Reif, and R.Wagner, "A refinement methodology for developing data-parallel applications," in Proc. EuroPar'96, Lecture Notes in Computer Science, Springer Verlag, 1996.

[34] S. J. Julier and J. K. Uhlmann, "A new extension of the Kalman filter to nonlinear systems," in Proc. AeroSense: 11th Int'l Symp. on Aerospace/Defence Sensing, Simulation and Controls, Orlando, Florida, 1997.

[35] T. Lefebvre, H. Bruyninckx, and J. D. Schutter, "Comment on "a new method for the nonlinear transformation of means and covariances in the filters and estimators"," IEEE Trans. AC, vol. AC-47, no. 8, pp. 1406-1409, 2002. 